\title{
New upper and lower bounds for randomized and quantum Local Search*
}

\author{
Shengyu Zhang ${ }^{\dagger}$
}

\begin{abstract}
Local Search problem, which finds a local minimum of a black-box function on a given graph, is of both practical and theoretical importance to combinatorial optimization, complexity theory and many other areas in theoretical computer science. In this paper, we study the problem in both the randomized and the quantum query models and give new lower and upper bound techniques in both models.

The lower bound technique works for any graph that contains a product graph as a subgraph. Applying it to the Boolean hypercube $\{0,1\}^{n}$ and the constant dimensional grids $[n]^{d}$, two particular product graphs that recently drew much attention, we get the following tight results:

$$
\begin{array}{ll}
R L S\left(\{0,1\}^{n}\right)=\Theta\left(2^{n / 2} n^{1 / 2}\right), & Q L S\left(\{0,1\}^{n}\right)=\Theta\left(2^{n / 3} n^{1 / 6}\right), \\
R L S\left([n]^{d}\right)=\Theta\left(n^{d / 2}\right) \text { for } d \geq 4, & Q L S\left([n]^{d}\right)=\Theta\left(n^{d / 3}\right) \text { for } d \geq 6 .
\end{array}
$$

Here $R L S(G)$ and $Q L S(G)$ are the randomized and quantum query complexities of Local Search on $G$, respectively. These improve the previous results by Aaronson [2], Ambainis (unpublished) and Santha and Szegedy[20].

Our new algorithms work well when the underlying graph expands slowly. As an application to $[n]^{2}$, a new quantum algorithm using $O\left(\sqrt{n}(\log \log n)^{1.5}\right)$ queries is given. This improves the previous best known upper bound of $O\left(n^{2 / 3}\right)$ (Aaronson, [2]), and implies that Local Search on grids exhibits different properties in low dimensions.
\end{abstract}

\footnotetext{
*This research was supported in part by NSF grants CCR-0310466 and CCF-0426582.

${ }^{\dagger}$ Computer Science Department, Princeton University, NJ 08544, USA. Email: szhang@cs.princeton.edu
} 


\section{Introduction}

Many important combinatorial optimization problems arising in both theory and practice are NPhard, which forces people to resort to heuristic searches in practice. One popular approach is Local Search, by which one first defines a neighborhood structure, then finds a solution that is locally optimal with respect to this neighborhood structure. In the past two decades, Local Search approach has been extensively developed and "has reinforced its position as a standard approach in combinatorial optimization" in practice [1]. Besides the practical applications, the problem also has many connections to the complexity theory, especially to the complexity classes PLS ${ }^{1}$ and TFNP 2. For example, the 2SAT-FLIP problem is Local Search on the Boolean hypercube graph $\{0,1\}^{n}$, with the objective function being the sum of the weights of the clauses that the truth assignment $x \in\{0,1\}^{n}$ satisfies. This problem is complete in PLS, implying that the Boolean hypercube $\{0,1\}^{n}$ has a central position in the studies of Local Search. Local Search is also related to physical systems including folding proteins and to quantum adiabatic algorithms [2]. We refer readers to the papers $[2,19,20]$ for more discussions and the book [3] for a comprehensive introduction.

Precisely, Local Search on an undirected graph $G=(V, E)$ is defined as follows. Given a function $f: V \rightarrow \mathbb{N}$, find a vertex $v \in V$ such that $f(v) \leq f(w)$ for all neighbors $w$ of $v$. A class of generic algorithms that has been widely used in practice is as follows: we first set out with an initial point $v \in V$, then repeatedly search a better/best neighbor until it reaches a local minimum. Though empirically this class of algorithms works very well in most applications, relatively few theoretical results are known about how good the generic algorithms are, especially for the randomized (and quantum) algorithms.

Among models for theoretical studies, the query model has drawn much attention $[2,4,5$, $16,17,20]$. In this model, $f$ is given by a black-box, i.e. $f(v)$ can be accessed by querying $v$. We only care about the number of queries made, and all other computations are free. If we are allowed to toss coins to decide the next query, then we have a randomized query algorithm. If we are allowed use quantum mechanics to query all the positions (and get corresponding answers) in superposition, then we have a quantum query algorithm. The deterministic, randomized and quantum query complexities are the minimum numbers of queries needed to compute the function by a deterministic, randomized and quantum query algorithm, respectively. We use $R L S(G)$ and $Q L S(G)$ to denote the randomized and quantum query complexities of Local Search on graph $G$, respectively. Previous upper bounds on a general $N$-vertex graph $G$ are $R L S(G)=O(\sqrt{N \delta})$ by Aldous [4] and $Q L S(G)=O\left(N^{1 / 3} \delta^{1 / 6}\right)$ by Aaronson [2], where $\delta$ is the maximum degree of $G$. Both algorithms actually fall into the category of generic algorithms mentioned above, with the initial point picked as a best one over a certain number of random samples. Immediately, two questions can be asked:

1. On what graphs are these simple algorithms optimal?

2. For other graphs, what better algorithms can we have?

Clearly the first one is a lower bound question and the second one is an upper bound question.

Previously for lower bounds, Aaronson [2] showed the following results on two special classes of graphs: the Boolean hypercube $\{0,1\}^{n}$ and the constant dimensional grid $[n]^{d}$ :

$$
\begin{array}{ll}
R L S\left(\{0,1\}^{n}\right)=\Omega\left(2^{n / 2} / n^{2}\right), & Q L S\left(\{0,1\}^{n}\right)=\Omega\left(2^{n / 4} / n\right) ; \\
R L S\left([n]^{d}\right)=\Omega\left(n^{d / 2-1} / \log n\right), & Q L S\left([n]^{d}\right)=\Omega\left(n^{d / 4-1 / 2} / \sqrt{\log n}\right) .
\end{array}
$$

It has also been shown that $Q L S\left([n]^{2}\right)=\Omega\left(n^{1 / 4}\right)$ by Santha and Szegedy in [20], besides their main result that the deterministic and the quantum query complexities of Local Search on any graph are polynomially related. However, the question

3. What are the true values of $Q L S$ and $R L S$ on $\{0,1\}^{n}$ and $[n]^{d}$ ?

remains an open problem, explicitly stated in an earlier version of [2] and also (partially) in [20].

\footnotetext{
${ }^{1}$ Polynomial Local Search, introduced by Johnson, Papadimitriou, and Yannakakis [14].

${ }^{2}$ The family of total function problems, introduced by Megiddo and Papadimitriou [18].
} 
In this paper, we answer questions 1 and 2 for large classes of graphs by giving both new lower and upper bound techniques for randomized and quantum query algorithms. As a consequence, we completely solve question 3 , except for a few small $d$ 's where our new bounds also significantly improve the old ones.

Our lower bound technique works for any graph that contains a product graph as a subgraph. For two graphs $G_{1}=\left(V_{1}, E_{1}\right)$ and $G_{2}=\left(V_{2}, E_{2}\right)$, their product $G_{1} \times G_{2}$ is the graph $G=(V, E)$ where $V=V_{1} \times V_{2}$ and

$$
E=\left\{\left(v_{1} \otimes v_{2}, v_{1}^{\prime} \otimes v_{2}\right):\left(v_{1}, v_{1}^{\prime}\right) \in E_{1}, v_{2} \in V_{2}\right\} \cup\left\{\left(v_{1} \otimes v_{2}, v_{1} \otimes v_{2}^{\prime}\right):\left(v_{2}, v_{2}^{\prime}\right) \in E_{2}, v_{1} \in V_{1}\right\}
$$

We will also use the notion of random walk on graphs to state the theorem. Given a graph $G=$ $(V, E)$, a random walk is a mapping $W: V \rightarrow 2^{V}$ where $W(u) \subseteq\{u\} \cup\{v:(u, v) \in E\}$. Intuitively, at each step the random walk $W$ goes from the current vertex $u$ to a uniformly random vertex in $W(u)$. The walk $W$ is regular if $|W(u)|=c$ for each $u \in V$. Denote by $p(u, v, t)$ the probability that the random walk starting at $u$ is at $v$ after exactly $t$ steps. Let $p_{t}=\max _{u, v} p(u, v, t)$. The following theorem is a special case of the general one (Theorem 9) in Section 3.

Theorem 1 Suppose $G$ contains the product graph $G_{1} \times G_{2}$ as a subgraph, and $L$ is the length of the longest self-avoiding path in $G_{2}$. Let $T=\lfloor L / 2\rfloor$, then for any regular random walk $W$ on $G_{1}$, we have

$$
R L S(G)=\Omega\left(\frac{T}{\sum_{t=1}^{T} p_{t}}\right), \quad Q L S(G)=\Omega\left(\frac{T}{\sum_{t=1}^{T} \sqrt{p_{t}}}\right) .
$$

The proof uses the quantum adversary method, which was originally proposed by Ambainis [7] and later generalized in different ways $[6,8,15,23]$. Recently Spalek and Szegedy made the picture clear by showing that all these generalizations are equivalent in power [21]. On the other hand, in proving a particular problem, some of the methods might be easier to apply than the others. In our case, the technique in [23], which generalizes the one in [6] slightly in the form though, turns out to work very well. Our proofs for the randomized lower bounds will use the relational adversary method, which was proposed by Aaronson [2] inspired by the quantum adversary method.

Both the quantum adversary method and the relational adversary method are parameterized by input sets and weight functions of input pairs. While previous works [2, 20] also use random walks on graphs, a key innovation that distinguishes our work from previous ones and yields better lower bounds is that we decompose the graph into two parts, the tensor product of which is the original graph. We perform the random walk only in one part, and perform a simple one-way walk in a self-avoiding path in the other part, which serves as a "clock" to record the number of steps taken by the random walk in the first part. The tensor product of these two walks is a random path in the original graph. A big advantage of adding a clock is that the "passing probability", the probability that the random path passes a vertex $v$ within $T$ steps, is now the "hitting probability", the probability that the random walk in the first graph hits $v$ after exactly $t$ steps, because the time elapses one-way and never comes back. The fact that the hitting probability is much smaller than the passing probability enables us to achieve the better lower bounds. Another advantage of the clock is that since the walk in the second part is self-avoiding, the resulting random path in the original graph is self-avoiding too, which makes the analysis much easier.

Applying it to the two graphs $\{0,1\}^{n}$ and $[n]^{d}$, we improve previous results and show tight bounds on both $R L S$ and $Q L S$ (except for a few cases in the low dimensional grids).

\section{Theorem 2}

$$
R L S\left(\{0,1\}^{n}\right)=\Theta\left(2^{n / 2} n^{1 / 2}\right), \quad Q L S\left(\{0,1\}^{n}\right)=\Theta\left(2^{n / 3} n^{1 / 6}\right) .
$$




\section{Theorem 3}

$$
R L S\left([n]^{d}\right)=\left\{\begin{array}{ll}
\Theta\left(n^{d / 2}\right) & \text { if } d \geq 4, \\
\Omega\left(\left(n^{3} / \log n\right)^{1 / 2}\right) & \text { if } d=3, \\
\Omega\left(n^{2 / 3}\right) & \text { if } d=2 .
\end{array} \quad Q L S\left([n]^{d}\right)= \begin{cases}\Theta\left(n^{d / 3}\right) & \text { if } d \geq 6, \\
\Omega\left(\left(n^{5} / \log n\right)^{1 / 3}\right) & \text { if } d=5, \\
\Omega\left(n^{6 / 5}\right) & \text { if } d=4, \\
\Omega\left(n^{3 / 4}\right) & \text { if } d=3, \\
\Omega\left(n^{2 / 5}\right) & \text { if } d=2 .\end{cases}\right.
$$

It is worth noting that to apply Theorem 1, we need to know not only the mixing time of the random walk in $G_{1}$, but also its behavior before mixing. So the applications are not simply using standard upper bounds on the mixing times, but involve heavy analysis on the whole mixing processes.

When proving Theorem 3 by Theorem 1, one difficulty arises: to decompose the grid $[n]^{d}$ into two parts $[n]^{m}$ and $[n]^{d-m}$, we implicitly require that $m$ is an integer. This gives us lower bounds weaker than Theorem 3, especially for low dimension cases. We get around this problem by cutting one of the $m$ dimensions into many blocks, and use different block to distinguish different time windows. Between adjacent blocks are pairwise disjoint path segments, which thus thread all the blocks into a very long one. Using this technique, we can apply Theorem 1 for any read-number dimension $m \leq d-1$.

In the second part of the paper, we consider upper bounds for Local Search. While the generic algorithms $[2,4]$ are simple and proven to be optimal for many graphs such as the ones mentioned above, they are far from optimal for some other graphs. For example, it is not hard to see an $O(\log N)$ deterministic algorithm for the line graph $G$. Therefore, a natural question is to characterize those graphs on which Local Search is easy. It turns out that the expansion speed plays a key role. For a graph $G=(V, E)$, the distance $l(u, v)$ between two vertices $u$ and $v$ is the length of the shortest path connecting them. (Here the length of a path is the number of edges on the path.) Let $c(k)=\max _{v \in V}|\{u: l(u, v) \leq k\}|$. Clearly, the smaller $c(k)$ is, the more slowly the graph expands. (Actually $c(k)$ is an upper bound of the standard definition of the expanding speed.) We say a graph is of polynomial growth if $c(k)=O\left(k^{\alpha}\right)$ for some constant $\alpha \geq 1$. As a special case of Theorem 12 in Section 5 , the following upper bounds for the graphs of polynomial growth.

Theorem 4 If $c(k)=O\left(k^{\alpha}\right)$ for some constant $\alpha \geq 1$, then

$$
R L S(G)=\left\{\begin{array}{ll}
O\left(d^{\alpha-1} \log \log d\right) & \text { if } \alpha>1, \\
O(\log d \log \log d) & \text { if } \alpha=1 .
\end{array} \quad Q L S(G)= \begin{cases}O\left(d^{\frac{\alpha-1}{2}}(\log \log d)^{1.5}\right) & \text { if } \alpha>1 \\
O(\log d \log \log d) & \text { if } \alpha=1\end{cases}\right.
$$

where $d$ is the diameter of the graph $G$.

As a special case, on the line graph we get $\alpha=1$ and hence $R L S=O(\log n \log \log n)$, which helps to explain why Local Search on the line graph is easy. Also, it immediately gives a new upper bound for $Q L S\left([n]^{2}\right)$ as follows. Together with Theorem 3, this implies that Local Search on grids exhibits different properties in low dimensions.

Theorem 5 QLS $\left([n]^{2}\right)=O\left(\sqrt{n}(\log \log n)^{1.5}\right)$

Other related results. After the preliminary version of this paper appeared, Verhoeven independently showed an upper bound in terms of the genus of the graph [22], giving an $O(\sqrt{n} \log \log n)$ quantum algorithm for $[n]^{2}$. There is also an unpublished result on $Q L S\left(\{0,1\}^{n}\right)$ : it is mentioned in [2] that Ambainis showed $Q L S\left(\{0,1\}^{n}\right)=\Omega\left(2^{n / 3} / n^{O(1)}\right){ }^{3}$

\footnotetext{
${ }^{3}$ Another unpublished result was mentioned in [20]: Verhoeven showed $R L S\left([n]^{2}\right)=\Omega\left(n^{1-\delta}\right)$ for any constant $\delta>0$. But according to Santha (personal communication), one of the two authors of [20], the proof was never written up and this question should be considered now to be still open.
} 


\section{Preliminaries and notations}

We use $[M]$ to denote the set $\{1,2, \ldots, M\}$. For an $n$-bit binary string $x=x_{0} \ldots x_{n-1} \in\{0,1\}^{n}$, let $x^{(i)}=x_{0} \ldots x_{i-1}\left(1-x_{i}\right) x_{i+1} \ldots x_{n-1}$ be the string obtained by flipping the coordinate $i$.

For graphs $G_{1}=\left(V_{1}, E_{1}\right)$ and $G_{2}=\left(V_{2}, E_{2}\right)$, we say that $G_{1}$ is a subgraph of $G_{2}$ if $V_{1} \subseteq V_{2}$ and $E_{1} \subseteq E_{2}$. Clearly, any local optimum in $G_{2}$ is also a local optimum in $G_{1}$ (but not the other way around in general), therefore any lower bound for $G_{1}$ is also a lower bound for $G_{2}$.

We let the variables $v_{1} \otimes v_{2}$ range over the set $V_{1} \times V_{2}$. There are various ways to define a product graph $G_{1} \times G_{2}=\left(V_{1} \times V_{2}, E\right)$ by different choices of $E$. Three possibilities are

1. $E=\left\{\left(v_{1} \otimes v_{2}, v_{1}^{\prime} \otimes v_{2}\right):\left(v_{1}, v_{1}^{\prime}\right) \in E_{1}, v_{2} \in V_{2}\right\} \cup\left\{\left(v_{1} \otimes v_{2}, v_{1} \otimes v_{2}^{\prime}\right):\left(v_{2}, v_{2}^{\prime}\right) \in E_{2}, v_{1} \in V_{1}\right\} ;$

2. $E^{\prime}=\left\{\left(v_{1} \otimes v_{2}, v_{1}^{\prime} \otimes v_{2}^{\prime}\right):\left(v_{1}, v_{1}^{\prime}\right) \in E_{1} \cup I_{V_{1}}\right.$ and $\left.\left(v_{2}, v_{2}^{\prime}\right) \in E_{2} \cup I_{V_{2}}\right\}-I_{V_{1} \times V_{2}}$, where $I_{V}=$ $\{(v, v): v \in V\}$

3. $E^{\prime \prime}=\left\{\left(v_{1} \otimes v_{2}, v_{1}^{\prime} \otimes v_{2}^{\prime}\right):\left(v_{1}, v_{1}^{\prime}\right) \in E_{1} \cup I_{V_{1}}\right.$ or $\left.\left(v_{2}, v_{2}^{\prime}\right) \in E_{2} \cup I_{V_{2}}\right\}-I_{V_{1} \times V_{2}}$.

It is clear that $E \subseteq E^{\prime} \subseteq E^{\prime \prime}$, and our lower bound theorem will use the first definition $E$, making the theorem as general as possible.

A path $X$ in a graph $G=(V, E)$ is a sequence $\left(v_{1}, \ldots, v_{l}\right)$ of vertices such that for any pair $\left(v_{i}, v_{i+1}\right)$ of vertices, either $v_{i}=v_{i+1}$ or $\left(v_{i}, v_{i+1}\right) \in E$. We use $\operatorname{set}(X)$ to denote the set of distinct vertices on path $X$. A path is self-avoiding if $v_{1}, \ldots, v_{l}$ are all distinct. The length of a path $\left(v_{1}, \ldots, v_{l}\right)$ is $l-1$. For two vertices $u, v \in V$, the distance $l_{G}(u, v)$ is the length of a shortest path from $u$ to $v$. The subscript $G$ may be omitted if no confusion is caused.

The $(k, l)$-hypercube $G_{k, l}=(V, E)$ where $V=[k]^{l}$ and whose edge set is $E=\{(u, v): \exists i \in$ $\{0, \ldots, l-1\}$, s.t. $\left|u_{i}-v_{i}\right|=1$, and $\left.u_{j}=v_{j}, \forall j \neq i\right\}$. Sometimes we abuse the notation by using $[k]^{l}$ to denote $G_{k, l}$. Note that both the Boolean hypercube and the constant dimension grid are special hypercubes. ${ }^{4}$

In an $N$-vertex graph $G=(V, E)$, a Hamilton path is a path $X=\left(v_{1}, \ldots, v_{N}\right)$ such that $\left(v_{i}, v_{i+1}\right) \in E$ for any $i \in[N-1]$ and $\operatorname{set}(X)=V$. It is easy to check by induction that every hypercube $[k]^{l}$ has a Hamilton path. Actually, for $l=1,[k]$ has a Hamilton path $(1, \ldots, k)$. Now suppose $[k]^{l}$ has a Hamilton path $P$; then a Hamilton path for $[k]^{l+1}$ can be constructed as follows. First fix the last coordinate to be 1 and go through $P$, then change the last coordinate to be 2 and go through $P$ in the reverse order, and then change the last coordinate to be 3 and go through $P$, and so on. For each $(k, l)$, let $\operatorname{HamPath}_{k, l}=\left(v_{1}, \ldots, v_{N}\right)$ be the Hamilton path constructed as above (where $N=k^{l}$ ), and we define the successor function $H_{k, l}\left(v_{i}\right)=v_{i+1}$ for $i \in[N-1]$.

As mentioned in Section 1, a deterministic query algorithm for a function $f: I^{n} \rightarrow[M]$ accesses the input $x \in I^{n}$ only by making queries in the form of " $x_{i}=$ ?". Each query has cost 1 , and all the other computation between queries are free. A randomized query algorithm is the same except that the algorithm can toss coins to decide which variable $x_{i}$ to ask next. The quantum query model, formally introduced in [9], has a working state in the form of $\sum_{i, a, z} \alpha_{i, a, z}|i, a, z\rangle$. A quantum query on the input $x$ proceeds as follows.

$$
\sum_{i, a, z} \alpha_{i, a, z}|i, a, z\rangle \rightarrow \sum_{i, a, z} \alpha_{i, a, z}\left|i, a \oplus x_{i}, z\right\rangle
$$

A $T$-query quantum query algorithm works as a sequence of operations

$$
U_{0} \rightarrow O_{x} \rightarrow U_{1} \rightarrow O_{x} \rightarrow \ldots \rightarrow U_{T-1} \rightarrow O_{x} \rightarrow U_{T}
$$

where $O_{x}$ is as defined above, and each $U_{t}$ does not depend on the input $x$. In both randomized and quantum query models, we can allow a double-sided small constant error probability. The deterministic, randomized and quantum query complexities, denoted by $D(f), R_{2}(f)$ and $Q_{2}(f)$, are the minimum numbers of queries we need to make in order to compute the function by a deterministic, randomized and quantum query algorithm, respectively. For more details on the query models and the corresponding query complexities, we refer to [10] as an excellent survey.

\footnotetext{
${ }^{4}$ Here we identify the Boolean hypercube $\{0,1\}^{n}$ and $G_{2, n}$ since they are isomorphic.
} 


\subsection{One quantum adversary method and the relational adversary method}

The quantum adversary method is one of the two powerful tools to prove lower bounds on quantum query complexity; see [13] for an comprehensive survey of this research area. In this paper, we will use the quantum adversary method proposed in [23]. The definition and theorem given here are a little more general than the original ones, but the proof remains unchanged.

Definition 1 Let $F: I^{N} \rightarrow[M]$ be an $N$-variate function. Let $R \subseteq I^{N} \times I^{N}$ be a relation such that $F(x) \neq F(y)$ for any $(x, y) \in R$. A weight scheme consists of three weight functions $w(x, y)>0$, $u(x, y, i)>0$ and $v(x, y, i)>0$ satisfying

$$
u(x, y, i) v(x, y, i) \geq w^{2}(x, y)
$$

for all $(x, y) \in R$ and $i \in[N]$ with $x_{i} \neq y_{i}$. We further put

$$
\begin{aligned}
w_{x} & =\sum_{y^{\prime}:\left(x, y^{\prime}\right) \in R} w\left(x, y^{\prime}\right), & w_{y} & =\sum_{x^{\prime}:\left(x^{\prime}, y\right) \in R} w\left(x^{\prime}, y\right) \\
u_{x, i} & =\sum_{y^{\prime}:\left(x, y^{\prime}\right) \in R, x_{i} \neq y_{i}^{\prime}} u\left(x, y^{\prime}, i\right), & v_{y, i} & =\sum_{x^{\prime}:\left(x^{\prime}, y\right) \in R, x_{i}^{\prime} \neq y_{i}} v\left(x^{\prime}, y, i\right) .
\end{aligned}
$$

Theorem 6 [Zhang, [23]] For any F, $R$ and any weight scheme $w, u, v$ as in Definition 1, we have

$$
Q_{2}(F)=\Omega\left(\min _{(x, y) \in R, i \in[N]: x_{i} \neq y_{i}} \sqrt{\frac{w_{x} w_{y}}{u_{x, i} v_{y, i}}}\right)
$$

In [2], Aaronson gives a nice technique to get a lower bound for randomized query complexity. We restate it using language similar to that of Theorem 6 .

Theorem 7 [Aaronson, [2]] Let $F: I^{N} \rightarrow[M]$ be an $N$-variate function. Let $R \subseteq I^{N} \times I^{N}$ be a relation such that $F(x) \neq F(y)$ for any $(x, y) \in R$. For any weight function $w: R \rightarrow \mathbb{R}^{+}$, we have

$$
R_{2}(F)=\Omega\left(\min _{(x, y) \in R, i \in[N], x_{i} \neq y_{i}} \max \left\{\frac{w_{x}}{w_{x, i}}, \frac{w_{y}}{w_{y, i}}\right\}\right)
$$

where

$$
w_{x, i}=\sum_{y^{\prime}:\left(x, y^{\prime}\right) \in R, x_{i} \neq y_{i}^{\prime}} w\left(x, y^{\prime}\right), \quad w_{y, i}=\sum_{x^{\prime}:\left(x^{\prime}, y\right) \in R, x_{i}^{\prime} \neq y_{i}} w\left(x^{\prime}, y\right) .
$$

Note that we can think of Theorem 7 as having a weight scheme too, but requiring that $u(x, y, i)=v(x, y, i)=w(x, y)$. This simple observation is used in the proof of Theorem 2 and 3.

\section{Lower bounds for Local Search on product graphs}

In this section we prove a theorem which is stronger than Theorem 1 due to a relaxation on the conditions of the random walk. Suppose we are given a graph $G=(V, E)$, a starting vertex $v_{0}$ and an assignment $W: V \times \mathbb{N} \rightarrow 2^{V}$ s.t. for each $u \in V$ and $t \in \mathbb{N}$, it holds that $W(u, t) \subseteq\{u\} \cup\{v$ : $(u, v) \in E\}$ and that $|W(u, t)|=c_{t}$ for some function $c$ of $t$. Intuitively, $W$ gives the candidates that the walk goes to for the next step, and the random walk $\left(G, v_{0}, W\right)$ on graph $G$ proceeds as follows. It starts at $v_{0}$, and at step $t \in \mathbb{N}$, it goes from the current vertex $v_{t-1}$ to a uniformly random vertex in $W\left(v_{t-1}, t\right)$. We say a path $\left(v_{0}, v_{1}, \ldots, v_{T}\right)$ is generated by the random walk if $v_{t} \in W\left(v_{t-1}, t\right)$ for all $t \in[T]$. Denote by $p\left(u, t_{1}, v, t_{2}\right)$ the probability that the random walk is at $v$ after step $t_{2}$ under the condition that the walk is at $u$ after step $t_{1}$. Let $p_{t}=\max _{u, v, t_{1}, t_{2}: t_{2}-t_{1}=t} p\left(u, t_{1}, v, t_{2}\right)$. For $\left(u, u^{\prime}\right) \in E$, let $q\left(u, u^{\prime}, t_{1}, v, t_{2}\right)$ be the probability that the walk is at $v$ after step $t_{2}$, under the conditions that 1) the walk is at $u$ after step $t_{1}$, and 2) the walk does not go to $u^{\prime}$ at step $t_{1}+1$. The following lemma on the relation of the two probabilities is obvious. 
Lemma 8 If $\left|W\left(u, t_{1}+1\right)\right|>1$, then $q\left(u, u^{\prime}, t_{1}, v, t_{2}\right) \leq 2 p\left(u, t_{1}, v, t_{2}\right)$.

Proof By considering the two cases of the step $t_{1}+1$ (going to $u^{\prime}$ or not), we have

$$
p\left(u, t_{1}, v, t_{2}\right)=\frac{1}{\left|W\left(u, t_{1}+1\right)\right|} p\left(u^{\prime}, t_{1}+1, v, t_{2}\right)+\left(1-\frac{1}{\left|W\left(u, t_{1}+1\right)\right|}\right) q\left(u, u^{\prime}, t_{1}, v, t_{2}\right) .
$$

Thus

$$
q\left(u, u^{\prime}, t_{1}, v, t_{2}\right) \leq p\left(u, t_{1}, v, t_{2}\right) /\left(1-\frac{1}{\left|W\left(u, t_{1}+1\right)\right|}\right) \leq 2 p\left(u, t_{1}, v, t_{2}\right)
$$

Theorem 9 Suppose $G$ contains $G^{w} \times G^{c}$ (for two arbitrary graphs $G^{w}$ and $G^{c}$ ) as a subgraph, and $L$ is the length of the longest self-avoiding path in $G^{c}$. Let $T=\lfloor L / 2\rfloor$, then for the random walk $\left(G^{w}, v_{0}^{w}, W\right)$ on $G^{w}$, we have

$$
R L S(G)=\Omega\left(\frac{T}{\sum_{t=1}^{T} p_{t}}\right), \quad Q L S(G)=\Omega\left(\frac{T}{\sum_{t=1}^{T} \sqrt{p_{t}}}\right) .
$$

Proof Without loss of generality, we assume $G=G^{w} \times G^{c}$, as Local Search on a subgraph is no harder than Local Search on the original graph. We shall construct a random walk on $G$ by the random walk $\left(G^{w}, v_{0}^{w}, W\right)$ on $G^{w}$ and a simple one-way walk on $G^{c}$. Starting from some fixed vertex in $G$, the walk proceeds by one step of walk in $G^{w}$ followed by two steps of walk in $G^{c}$. (We perform two steps of walk in $G^{c}$ mainly for some technical reasons, and this is where the factor of 2 in definition $T=\lfloor L / 2\rfloor$ comes from.) Precisely, fix a self-avoiding path $\left(z_{0,0}^{c}, z_{1,0}^{c}, z_{1,1}^{c}, z_{2,1}^{c}, z_{2,2}^{c}, \ldots, z_{T, T-1}^{c}, z_{T, T}^{c}\right)$ of length $2 T$ in $G^{c}$. Let the set $P$ contain all the paths $X=\left(x_{0}^{w} \otimes z_{0,0}^{c}, x_{1}^{w} \otimes z_{0,0}^{c}, x_{1}^{w} \otimes z_{1,0}^{c}, x_{1}^{w} \otimes z_{1,1}^{c}, \ldots, x_{T}^{w} \otimes z_{T-1, T-1}^{c}, x_{T}^{w} \otimes z_{T, T-1}^{c}, x_{T}^{w} \otimes z_{T, T}^{c}\right)$ in $G$ such that $x_{0}^{w}=v_{0}^{w}$ and $\left(x_{0}^{w}, x_{1}^{w}, \ldots, x_{T}^{w}\right)$ is a path generated by the random walk $\left(G^{w}, v_{0}^{w}, W\right)$. Define a problem $\operatorname{PATH}_{P}$ : given a path $X \in P$, find the end point $x_{T}^{w} \otimes z_{T, T}^{c}$. To access $X$, we can ask whether $v \in \operatorname{set}(X)$ for any vertex $v \in V$, and an oracle $O$ will give us the Yes/No answer. ${ }^{5}$ The following claim says that the $\mathrm{PATH}_{P}$ problem is not much harder than the Local Search problem.

Claim $1 R_{2}\left(\mathrm{PATH}_{P}\right) \leq 2 R L S(G), \quad Q_{2}\left(\mathrm{PATH}_{P}\right) \leq 2 Q L S(G)$.

Proof Suppose we have an $Q$-query randomized or quantum algorithm $\mathcal{A}$ for Local Search, we shall give a $2 Q$ corresponding algorithm $\mathcal{B}$ for $\operatorname{PATH}_{P}$. For any path $X \in P$, we define a function $f_{X}$ essentially in the same way as Aaronson did in [2]: for each vertex $v \in G$, let

$$
f_{X}(v)=\left\{\begin{array}{ll}
l_{G}\left(v, x_{0}^{w} \otimes z_{0,0}^{c}\right)+3 T & \text { if } v \notin \operatorname{set}(X) \\
3(T-k) & \text { if } v=x_{k}^{w} \otimes z_{k, k}^{c} \\
3(T-k)-1 & \text { if } v=x_{k+1}^{w} \otimes z_{k, k}^{c} \neq x_{k}^{w} \otimes z_{k, k}^{c} \\
3(T-k)-2 & \text { if } v=x_{k+1}^{w} \otimes z_{k+1, k}^{c}
\end{array} .\right.
$$

It is easy to verify that the only local minimum is $x_{T}^{w} \otimes z_{T, T}^{c}$.

Given an oracle $O$ and an input $X$ of the PATH problem, $\mathcal{B}$ simulates $\mathcal{A}$ to find the local minimum of $f_{X}$, which is also the end point of $X$. Whenever $\mathcal{A}$ needs to make a query on $v$ to get $f_{X}(v)$, $\mathcal{B}$ asks $O$ whether $v \in \operatorname{set}(X)$. If $v \notin \operatorname{set}(X)$, then $f_{X}(v)=l_{G}\left(v, x_{0}^{w} \otimes z_{0,0}^{c}\right)+3 T$; otherwise, $v=x^{w} \otimes z_{k+1, k}^{c}$ or $v=x^{w} \otimes z_{k, k}^{c}$ for some $x^{w} \in V^{w}$ and $k$. Note that $k$ is known for any given vertex $v$. So if $v=x^{w} \otimes z_{k+1, k}^{c}$, then $x^{w}=x_{k+1}^{w}$ and thus $f_{X}(v)=3(T-k)-2$. Now consider

\footnotetext{
${ }^{5}$ Note that it is actually an oracle for the following function $g:\{0,1\}^{n} \rightarrow\{0,1\}$, with $g(x)=1$ if and only if $x \in \operatorname{set}(X)$. So strictly speaking, an input of $\mathrm{PATH}_{P}$ should be specified as $\operatorname{set}(X)$ rather than $X$, because in general, it is possible that $X \neq Y$ but $\operatorname{set}(X)=\operatorname{set}(Y)$. For our problem, however, it is easy to check that for any $X, Y \in P$, it holds that $X=Y \Leftrightarrow \operatorname{set}(X)=\operatorname{set}(Y)$. Indeed, if $X \neq Y$, suppose the first diverging place is $k$, i.e. $x_{k-1}^{w}=y_{k-1}^{w}$, but $x_{k}^{w} \neq y_{k}^{w}$. Then $Y$ will never pass $x_{k}^{w} \otimes z_{k, k-1}^{c}$ because the clock immediately ticks and the time always advances forward. (Or more rigorously, the only point that $Y$ passes through $z_{k, k-1}^{c}$ is $y_{k}^{w} \otimes z_{k, k-1}^{c}$. Since $y_{k}^{w} \neq x_{k}^{w}, x_{k}^{w} \otimes z_{k, k-1}^{c} \notin \operatorname{set}(Y)$.)
} 
the case that $v=x^{w} \otimes z_{k, k}^{c}$. If $k=0$, then let $f_{X}(v)=3 T$ if $v=x_{0}^{w} \otimes z_{0,0}^{c}$ and $f_{X}(v)=3 T-1$ otherwise. If $k \geq 1$, then $\mathcal{B}$ asks $O$ whether $x^{w} \otimes z_{k, k-1}^{c} \in \operatorname{set}(X)$. If yes, then $v=x_{k}^{w} \otimes z_{k, k}^{c}$ and thus $f_{X}(v)=3(T-k)$; if no, then $v=x_{k+1}^{w} \otimes z_{k, k}^{c} \neq x_{k}^{w} \otimes z_{k, k}^{c}$ and thus $f_{X}(v)=3(T-k)-1$. Therefore, at most 2 queries on $O$ can simulate one query on $f_{X}$, so we have a $2 Q$ algorithm for $\mathrm{PATH}_{P}$ in both randomized and quantum cases.

(Continue the proof of Theorem 9) By the claim, it is sufficient to prove lower bounds for $\mathrm{PATH}_{P}$. We define a relation $R_{P}$ as follows.

$$
R_{P}=\{(X, Y): X \in P, Y \in P, X \text { and } Y \text { has different end points }\} .
$$

For any pair $(X, Y) \in R_{P}$, where $X=\left(x_{0}^{w} \otimes z_{0,0}^{c}, x_{1}^{w} \otimes z_{0,0}^{c}, x_{1}^{w} \otimes z_{1,0}^{c}, x_{1}^{w} \otimes z_{1,1}^{c}, \ldots, x_{T}^{w} \otimes z_{T-1, T-1}^{c}, x_{T}^{w} \otimes\right.$ $\left.z_{T, T-1}^{c}, x_{T}^{w} \otimes z_{T, T}^{c}\right)$ and $Y=\left(y_{0}^{w} \otimes z_{0,0}^{c}, y_{1}^{w} \otimes z_{0,0}^{c}, y_{1}^{w} \otimes z_{1,0}^{c}, y_{1}^{w} \otimes z_{1,1}^{c}, \ldots, y_{T}^{w} \otimes z_{T-1, T-1}^{c}, y_{T}^{w} \otimes z_{T, T-1}^{c}, y_{T}^{w} \otimes\right.$ $z_{T, T}^{c}$ ), we write $X \wedge Y=k$ if $x_{0}^{w}=y_{0}^{w}, \ldots, x_{k-1}^{w}=y_{k-1}^{w}$ but $x_{k}^{w} \neq y_{k}^{w}$. Intuitively, $X \wedge Y=k$ if $k$ is the place that the paths $X$ and $Y$ diverge for the first time. Note that if $X \wedge Y=k$, then $x_{k}^{w}, y_{k}^{w} \in W\left(x_{k-1}^{w}, k\right)$ and thus $\left|W\left(x_{k-1}^{w}, k\right)\right| \geq 2$. By Lemma 8, this implies that $q\left(x_{k-1}^{w}, x_{k}^{w}, k-\right.$ $\left.1, v^{w}, j\right) \leq 2 p_{j-k+1}$.

We choose the weight functions in Theorem 6 by letting

$$
\begin{aligned}
w(X, Y) & =1 /\left|\left\{Y^{\prime} \in P: Y^{\prime} \wedge X=k\right\}\right| \\
& =1 /\left|\left\{X^{\prime} \in P: X^{\prime} \wedge Y=k\right\}\right| \\
& =1 /\left[\left(c_{k}-1\right) c_{k+1} \ldots c_{T}\right] .
\end{aligned}
$$

To calculate $w_{X}=\sum_{Y^{\prime}:\left(X, Y^{\prime}\right) \in R_{P}} w\left(X, Y^{\prime}\right)$, we group those $Y^{\prime}$ that diverge from $X$ at the same place $k^{\prime}$ :

$$
\begin{aligned}
w_{X} & =\sum_{k^{\prime}=1}^{T} \sum_{\substack{Y^{\prime}:\left(X, Y^{\prime}\right) \in R_{P} \\
X \wedge Y^{\prime}=k^{\prime}}} w\left(X, Y^{\prime}\right) \\
& =\sum_{k^{\prime}=1}^{T} \sum_{\substack{Y^{\prime}:\left(X, Y^{\prime}\right) \in R_{P} \\
X \wedge Y^{\prime}=k^{\prime}}} \frac{1}{\left|\left\{Y^{\prime} \in P: Y^{\prime} \wedge X=k^{\prime}\right\}\right|} \\
& =\sum_{k^{\prime}=1}^{T} \operatorname{Pr}_{Y^{\prime}}\left[\left(X, Y^{\prime}\right) \in R_{P} \mid Y^{\prime} \wedge X=k^{\prime}\right] \\
& =\sum_{k^{\prime}=1}^{T} \operatorname{Pr}_{Y^{\prime}}\left[\left(y^{\prime}\right)_{T}^{w} \neq x_{T}^{w} \mid Y^{\prime} \wedge X=k^{\prime}\right]
\end{aligned}
$$

Here Equality (25) holds because all paths diverging from $X$ firstly at $k^{\prime}$ have the same probability $1 /\left[\left(c_{k^{\prime}}-1\right) c_{k^{\prime}} \ldots c_{T}\right]$. Also note that the probability in the last equality is nothing but $1-q\left(x_{k^{\prime}-1}^{w}, x_{k^{\prime}}^{w}, k^{\prime}-1, x_{T}^{w}, T\right)$, which is at least $1-2 p_{T-k^{\prime}+1}$. So we have

$$
w_{X} \geq T-2 \sum_{k^{\prime}=1}^{T} p_{T-k^{\prime}+1}=T-2 \sum_{t=1}^{T} p_{t} .
$$

And similarly, we have $w_{Y} \geq T-2 \sum_{t=1}^{T} p_{t}$ too.

Now we describe $u(X, Y, i)$ and $v(X, Y, i)$, where $i$ is a point $x_{j+r}^{w} \otimes z_{j+s, j}^{c} \in \operatorname{set}(X)-\operatorname{set}(Y)$ or $y_{j+r}^{w} \otimes z_{j+s, j}^{c} \in \operatorname{set}(Y)-\operatorname{set}(X)$. Here $(r, s) \in\{(0,0),(1,0),(1,1)\}$, and $0 \leq j \leq j+r \leq T$. Let

$$
\begin{array}{ll}
u\left(X, Y, x_{j+r}^{w} \otimes z_{j+s, j}^{c}\right)=a_{k, j, r, s} w(X, Y), & u\left(X, Y, y_{j+r}^{w} \otimes z_{j+s, j}^{c}\right)=b_{k, j, r, s} w(X, Y), \\
v\left(X, Y, x_{j+r}^{w} \otimes z_{j+s, j}^{c}\right)=b_{k, j, r, s} w(X, Y), & v\left(X, Y, y_{j+r}^{w} \otimes z_{j+s, j}^{c}\right)=a_{k, j, r, s} w(X, Y),
\end{array}
$$

where $a_{k, j, r, s}$ and $b_{k, j, r, s}$ will be given later (satisfying $a_{k, j, r, s} b_{k, j, r, s}=1$, which makes $u, v, w$ really a weight scheme). We shall calculate $u_{X, i}$ and $v_{Y, i}$ for $i=x_{j+r}^{w} \otimes z_{j+s, j}^{c} \in \operatorname{set}(X)-\operatorname{set}(Y)$; the 
other case $i=y_{j+r}^{w} \otimes z_{j+s, j}^{c}$ is symmetric. Note that if $x_{j+r}^{w} \otimes z_{j+s, j}^{c} \notin \operatorname{set}\left(Y^{\prime}\right)$ and $X \wedge Y^{\prime}=k^{\prime}$, then $k^{\prime} \leq j+r$.

$$
\begin{aligned}
u_{X, x_{j+r}^{w} \otimes z_{j+s, j}^{c}} & =\sum_{k^{\prime}=1}^{j+r} \sum_{\substack{Y^{\prime}:\left(X, Y^{\prime}\right) \in R_{P}, X \wedge Y^{\prime}=k^{\prime} \\
x_{j+r}^{w} \otimes z_{j+s, j}^{c} \notin \operatorname{set}\left(Y^{\prime}\right)}} a_{k^{\prime}, j, r, s} w\left(X, Y^{\prime}\right) \\
& \leq \sum_{k^{\prime}=1}^{j+r} \sum_{Y^{\prime}: X \wedge Y^{\prime}=k^{\prime}} a_{k^{\prime}, j, r, s} w\left(X, Y^{\prime}\right) \\
& =\sum_{k^{\prime}=1}^{j+r} a_{k^{\prime}, j, r, s}
\end{aligned}
$$

The computation for $v_{Y, x_{j+r}^{w}} \otimes z_{j+s, j}^{c}$ is a little more complicated. By definition,

$$
\begin{aligned}
v_{Y, x_{j+r}^{w}}^{w} \otimes z_{j+s, j}^{c} & =\sum_{k^{\prime}=1}^{j+r} \sum_{\substack{X^{\prime}:\left(X^{\prime}, Y\right) \in R_{P}, X^{\prime} \wedge Y=k^{\prime}, x_{j+r}^{w} \otimes z_{j+s, j}^{c} \in \operatorname{set}\left(X^{\prime}\right)}} b_{k^{\prime}, j, r, s} w\left(X^{\prime}, Y\right) \\
& \leq \sum_{k^{\prime}=1}^{j+r} \sum_{\substack{X^{\prime}: X^{\prime} \wedge Y=k^{\prime}, x_{j+r}^{w} \otimes z_{j+s, j}^{c} \in \operatorname{set}\left(X^{\prime}\right)}} b_{k^{\prime}, j, r, s} w\left(X^{\prime}, Y\right) \\
& =\sum_{k^{\prime}=1}^{j+r} b_{k^{\prime}, j, r, s} \operatorname{Pr}_{X^{\prime}}\left[x_{j+r}^{w} \otimes z_{j+s, j}^{c} \in \operatorname{set}\left(X^{\prime}\right) \mid X^{\prime} \wedge Y=k^{\prime}\right]
\end{aligned}
$$

We can see that by adding the clock, the passing probability $\operatorname{Pr}_{X^{\prime}}\left[x_{j+r}^{w} \otimes z_{j+s, j}^{c} \in \operatorname{set}\left(X^{\prime}\right) \mid X^{\prime} \wedge Y=\right.$ $\left.k^{\prime}\right]$ is roughly the hitting probability $q\left(y_{k^{\prime}-1}^{w}, y_{k^{\prime}}^{w}, k^{\prime}-1, x_{j+r}^{w}, j\right)+q\left(y_{k^{\prime}-1}^{w}, y_{k^{\prime}}^{w}, k^{\prime}-1, x_{j+r}^{w}, j+1\right)$ except for some corner cases. To be more precise, define

$$
\text { Bound }_{k^{\prime}, j, r, s}=2 p_{j-k^{\prime}+2} \cdot \lambda[s=1 \text { OR } j<T]+2 p_{j-k^{\prime}+1} \cdot \lambda\left[s=0 \text { AND }\left(k^{\prime} \leq j \text { OR } r=0\right)\right]
$$

where the Boolean function $\lambda[\phi]=1$ if $\phi$ is true and 0 otherwise. Then

Claim $2 \quad \operatorname{Pr}_{X^{\prime}}\left[x_{j+r}^{w} \otimes z_{j+s, j}^{c} \in \operatorname{set}\left(X^{\prime}\right) \mid X^{\prime} \wedge Y=k^{\prime}\right] \leq$ Bound $_{k^{\prime}, j, r, s}$.

Proof We study the probability $\operatorname{Pr}_{X^{\prime}}\left[x_{j+r}^{w} \otimes z_{j+s, j}^{c} \in \operatorname{set}\left(X^{\prime}\right) \mid X^{\prime} \wedge Y=k^{\prime}\right]$ case by case. If $s=1$, then $r=1$, and $x_{j+1}^{w} \otimes z_{j+1, j}^{c} \in \operatorname{set}\left(X^{\prime}\right)$ if and only if $x_{j+1}^{w}=\left(x^{\prime}\right)_{j+1}^{w}$. So

$$
\operatorname{Pr}_{X^{\prime}}\left[x_{j+r}^{w} \otimes z_{j+s, j}^{c} \in \operatorname{set}\left(X^{\prime}\right) \mid X^{\prime} \wedge Y=k^{\prime}\right]=q\left(y_{k^{\prime}-1}^{w}, y_{k^{\prime}}^{w}, k^{\prime}-1, x_{j+1}^{w}, j+1\right) \leq 2 p_{j-k^{\prime}+2}
$$

by Lemma 8. If $s=0$, then $x_{j+r}^{w} \otimes z_{j, j}^{c} \in \operatorname{set}\left(X^{\prime}\right)$ if and only if " $x_{j+r}^{w}=\left(x^{\prime}\right)_{j}^{w}$ or $x_{j+r}^{w}=\left(x^{\prime}\right)_{j+1}^{w}$ ". Also note that

$$
\operatorname{Pr}_{X^{\prime}}\left[x_{j+r}^{w}=\left(x^{\prime}\right)_{j}^{w} \mid X^{\prime} \wedge Y=k^{\prime}\right]=q\left(y_{k^{\prime}-1}^{w}, y_{k^{\prime}}^{w}, k^{\prime}-1, x_{j+r}^{w}, j\right)
$$

unless $k^{\prime}=j+1$ and $r=1$, in which case $\operatorname{Pr}_{X^{\prime}}\left[x_{j+r}^{w}=\left(x^{\prime}\right)_{j}^{w} \mid X^{\prime} \wedge Y=k^{\prime}\right]=0$ because $x_{j+1}^{w} \otimes z_{j, j}^{c} \notin$ $\operatorname{set}(Y)$ but $\left(x^{\prime}\right)_{j}^{w} \otimes z_{j, j}^{c}=y_{j}^{w} \otimes z_{j, j}^{c} \in \operatorname{set}(Y)$. The other probability

$$
\operatorname{Pr}_{X^{\prime}}\left[x_{j+r}^{w}=\left(x^{\prime}\right)_{j+1}^{w} \mid X^{\prime} \wedge Y=k^{\prime}\right]=\left\{\begin{array}{ll}
q\left(y_{k^{\prime}-1}^{w}, y_{k^{\prime}}^{w}, k^{\prime}-1, x_{j+r}^{w}, j+1\right) & \text { if } j \leq T-1 \\
0 & \text { if } j=T
\end{array} .\right.
$$

Putting all cases together, we get the desired result.

(Continue the proof of Theorem 9) The claim implies that

$$
v_{Y, x_{j+r}^{w}} \otimes z_{j+s, j}^{c} \leq \sum_{k^{\prime}=1}^{j+r} b_{k^{\prime}, j, r, s} \text { Bound }_{k^{\prime}, j, r, s} .
$$


The symmetric case of $u(X, Y, i)$ and $v(X, Y, i)$ where $i$ is a point $y_{j+r}^{w} \otimes z_{j+s, j}^{c} \in \operatorname{set}(Y)-$ $\operatorname{set}(X)$ can be dealt with in the same way, yielding $u_{X, y_{j+r}^{w} \otimes z_{j+s, j}^{c}} \leq \sum_{k^{\prime}=1}^{j+r} b_{k^{\prime}, j, r, s} B_{o u n d} d_{k^{\prime}, j, r, s}$ and $v_{Y, y_{j+r}^{w}}^{w} \otimes z_{j+s, j}^{c} \leq \sum_{k^{\prime}=1}^{j+r} a_{k^{\prime}, j, r, s}$.

By the definition of Bound $k_{k^{\prime}, j, r, s}$, it holds for any $(j, r, s)$ that

$$
\sum_{k^{\prime}=1}^{j+r} \text { Bound }_{k^{\prime}, j, r, s} \leq 4 \sum_{t=1}^{T} p_{t} \quad \text { and } \quad \sum_{k^{\prime}=1}^{j+r} \sqrt{\text { Bound }_{k^{\prime}, j, r, s}} \leq 4 \sum_{t=1}^{T} \sqrt{p_{t}} .
$$

Now for the randomized lower bound, $a_{k^{\prime}, j, r, s}=b_{k^{\prime}, j, r, s}=1$.

$$
R L S(G)=\Omega\left(\min _{j, r, s} \max \left\{\frac{T-2 \sum_{t=1}^{T} p_{t}}{j+r}, \frac{T-2 \sum_{t=1}^{T} p_{t}}{\sum_{k^{\prime}=1}^{j+r} \text { Bound }_{k^{\prime}, j, r, s}}\right\}\right)=\Omega\left(\frac{T}{\sum_{t=1}^{T} p_{t}}\right) .
$$

For the quantum lower bound, pick $a_{k^{\prime}, j, r, s}=\sqrt{\operatorname{Bound}_{k^{\prime}, j, r, s}}$, and $b_{k^{\prime}, j, r, s}=1 / \sqrt{\text { Bound }_{k^{\prime}, j, r, s}}$. Then

$$
Q L S(G)=\Omega\left(\min _{j, r, s} \sqrt{\frac{\left(T-2 \sum_{t=1}^{T} p_{t}\right)\left(T-2 \sum_{t=1}^{T} p_{t}\right)}{\left(\sum_{k^{\prime}=1}^{j+r} \sqrt{\text { Bound }_{k^{\prime}, j, r, s}}\right)\left(\sum_{k^{\prime}=1}^{j+r} \sqrt{\text { Bound }_{k^{\prime}, j, r, s}}\right)}}\right)=\Omega\left(\frac{T}{\sum_{t=1}^{T} \sqrt{p_{t}}}\right)
$$

This completes the proof of Theorem 9 .

\section{Applications to the two special graphs}

In this section, we will apply Theorem 9 to the two special graphs. Note that in both cases, the probability $p_{t}$ is not easy to upper bound. Also note that we need not only to pick the random walk, but also the way to decompose the graph.

\subsection{Lower bounds for Local Search on the Boolean Hypercube}

To apply Theorem 9 to $\{0,1\}^{n}$, we decompose the whole graph into the two parts $\{0,1\}^{m}$ and $\{0,1\}^{n-m}$, where $m$ is to be decided later (and to be taken different values for randomized and quantum lower bounds). Pick the random walk $\left(\{0,1\}^{m}, v_{0}^{w}, W\right)$, where $v_{0}^{w}=0^{m} \in\{0,1\}^{m}$ and $W(x, t)=\left\{x^{(i)}: i \in\{0, \ldots, m-1\}\right\}$ for each vertex $x=x_{0} \ldots x_{m-1} \in\{0,1\}^{m}$ and each $t \in \mathbb{N}$. Finally, note that the longest self-avoiding path of the graph $\{0,1\}^{n-m}$ is a Hamilton path with length $L=2^{n-m}-1$.

The following bounds on $p_{t}$ are rather loose for $10<t \leq m^{2}$ but sufficient for our purpose. The proof of the lemma uses some techniques in generating functions and Fourier analysis.

Lemma 10 For any $t \in \mathbb{N}$, we have

$$
p_{t}= \begin{cases}O\left(m^{-\lceil t / 2\rceil}\right) & \text { if } t \leq 10 \\ O\left(m^{-5}\right) & \text { if } 10<t \leq m^{2} \\ O\left(2^{-m}\right) & \text { if } t>m^{2}\end{cases}
$$

Proof Consider that we put $t$ balls randomly into $m$ bins one by one. The $j$-th ball goes into the $i_{j}$-th bin. Denote by $n_{i}$ the total number of balls in the $i$-th bin. We write $n_{i} \equiv b_{i}$ if $b_{i}=$ $n_{i} \bmod 2$. We say that $\left(i_{1}, \ldots, i_{t}\right)$ generates the parity sequence $\left(b_{1}, \ldots, b_{m}\right)$, or simply $\left(i_{1}, \ldots, i_{t}\right)$ generates $\left(b_{1}, \ldots, b_{m}\right)$, if $n_{i} \equiv b_{i}$ for all $i \in[m]$. For $b_{1} \ldots b_{m} \in\{0,1\}^{m}$, denote by $p^{(t)}\left[b_{1}, \ldots, b_{m}\right]$ the probability that $n_{i} \equiv b_{i}, \forall i \in[m]$. Let $p^{(t)}=\max _{b_{1}, \ldots, b_{m}} p^{(t)}\left[b_{1}, \ldots, b_{m}\right]$. It is easy to see that $p^{(t)}=p_{t}$ in Lemma 10, so it is enough to prove the same bounds in Lemma 10 for $p^{(t)}$. 
We start with several simple observations. First, we assume that $t$ and $\sum_{i=1}^{m} b_{i}$ have the same parity, because otherwise the probability is 0 and the lemma holds trivially. Second, by the symmetry, any permutation of $b_{1}, \ldots, b_{m}$ does not change $p^{(t)}\left[\left(b_{1}, \ldots, b_{m}\right)\right]$. Third, $p^{(t)}\left[\left(b_{1}, \ldots, b_{m}\right)\right]$ decreases if we replace two 1's in $b_{1}, \ldots, b_{m}$ by two 0 's. Precisely, if we have two $b_{i}$ 's being 1 , say $b_{1}=b_{2}=1$, then $p^{(t)}\left[\left(b_{1}, \ldots, b_{m}\right)\right]<p^{(t)}\left[\left(0,0, b_{3}, \ldots, b_{m}\right)\right]$. In fact, note that

$$
\begin{aligned}
p^{(t)}\left[\left(b_{1}, \ldots, b_{m}\right)\right] & =\frac{1}{m^{t}} \sum_{\substack{n_{1}+\ldots+n_{m}=t \\
n_{i}=b_{i}, i \in[m]}} \frac{t !}{n_{1} ! \ldots n_{m} !} \\
& =\frac{1}{m^{t}} \sum_{\substack{n_{3}+\ldots, n_{m} \leq t \\
n_{i}=b_{i}, i=3, \ldots, m}}\left(\frac{t !}{\left(n_{1}+n_{2}\right) ! n_{3} ! \ldots n_{m} !} \sum_{\substack{n_{1}+n_{2}=t-n_{3}-\ldots-n_{m} \\
n_{i} \equiv b_{i}, i=1,2}} \frac{\left(n_{1}+n_{2}\right) !}{n_{1} ! n_{2} !}\right)
\end{aligned}
$$

where as usual, let $0 !=1$. If $n_{3}+\ldots+n_{m}<t$, then

$$
\sum_{\substack{n_{1}+n_{2}=t-n_{3}-\ldots-n_{m} \\ n_{i}=1, i=1,2}} \frac{\left(n_{1}+n_{2}\right) !}{n_{1} ! n_{2} !}=\sum_{\substack{n_{1}+n_{2}=t-n_{3}-\ldots, n_{m} \\ n_{i}=0, i=1,2}} \frac{\left(n_{1}+n_{2}\right) !}{n_{1} ! n_{2} !}
$$

If $n_{3}+\ldots+n_{m}=t$, then the only possible $\left(n_{1}, n_{2}\right)$ is $(0,0)$, so

$$
\sum_{\substack{n_{1}+n_{2}=t-n_{3}-\ldots-n_{m} \\ n_{i} \equiv 1, i=1,2}} \frac{\left(n_{1}+n_{2}\right) !}{n_{1} ! n_{2} !}=0, \quad \sum_{\substack{n_{1}+n_{2}=t-n_{3}-\ldots-n_{m} \\ n_{i}=0, i=1,2}} \frac{\left(n_{1}+n_{2}\right) !}{n_{1} ! n_{2} !}=1 .
$$

Thus $p^{(t)}\left[\left(1,1, b_{3}, \ldots, b_{m}\right)\right]<p^{(t)}\left[\left(0,0, b_{3}, \ldots, b_{m}\right)\right]$.

By these observations, it is sufficient to prove the lemma for the case $p^{(t)}[(0, \ldots, 0)]$ if $t$ is even, and for the case $p^{(t)}[(1,0, \ldots, 0)]$ if $t$ is odd. Note that if $t$ is even, then

$$
p^{(t)}[(0, \ldots, 0)]=\sum_{i=1}^{m} \operatorname{Pr}\left[i_{1}=i\right] \operatorname{Pr}\left[\left(i_{2}, \ldots, i_{t}\right) \text { generates }\left(e_{i}\right)\right]
$$

where $e_{i}$ is the $m$-long vector with only coordinate $i$ being 1 and all other coordinates being 0 . By symmetry, $p^{(t-1)}\left[e_{1}\right]=\ldots=p^{(t-1)}\left[e_{m}\right]$, thus $p^{(t)}[(0, \ldots 0)]=p^{(t-1)}\left[e_{1}\right]=p^{(t-1)}[1,0, \ldots, 0]$. Therefore, it is enough to show the lemma for even $t$.

We now express $p^{(t)}[0, \ldots, 0]$ in two ways. One is to prove the first case $(t \leq 10)$ in the lemma, and the other is for the second case $\left(10<t \leq m^{2}\right)$ and the third case $\left(t>m^{2}\right)$ in the lemma.

To avoid confusion, we write the number $m$ of bins explicitly as subscript: $p_{m}^{(t)}\left[b_{1}, \ldots, b_{m}\right]$. We consider which bin(s) the first two balls are put into.

$$
\begin{aligned}
p_{m}^{(t)}[0, \ldots, 0] & =\operatorname{Pr}\left[i_{1}=i_{2}\right] p_{m}^{(t-2)}[0, \ldots, 0]+\operatorname{Pr}\left[i_{1} \neq i_{2}\right] p_{m}^{(t-2)}[1,1,0, \ldots, 0] \\
& =\frac{1}{m} p_{m}^{(t-2)}[0, \ldots, 0]+\frac{m-1}{m} p_{m}^{(t-2)}[1,1,0, \ldots, 0]
\end{aligned}
$$

To compute $p_{m}^{(t-2)}[1,1,0, \ldots, 0]$, we consider how to put $(t-2)$ balls in $m$ bins. By the analysis of the third observation above, we know that

$$
\begin{aligned}
& p_{m}^{(t-2)}[0, \ldots, 0]-p_{m}^{(t-2)}[1,1,0, \ldots, 0] \\
= & \operatorname{Pr}\left[n_{1}=n_{2}=0, n_{3} \equiv 0, \ldots, n_{m} \equiv 0\right] \\
= & \operatorname{Pr}\left[n_{1}=n_{2}=0\right] \operatorname{Pr}\left[n_{3} \equiv 0, \ldots, n_{m} \equiv 0 \mid n_{1}=n_{2}=0\right] \\
= & \left(\frac{m-2}{m}\right)^{t-2} p_{m-2}^{(t-2)}[0, \ldots, 0]
\end{aligned}
$$

Therefore,

$$
p_{m}^{(t)}[0, \ldots, 0]=\frac{1}{m} p_{m}^{(t-2)}[0, \ldots, 0]-\frac{m-1}{m}\left(\frac{m-2}{m}\right)^{t-2} p_{m-2}^{(t-2)}[0, \ldots, 0]
$$


Now using the above recursive formula and the base case $p_{m}^{(2)}[0, \ldots, 0]=1 / m$, it is easy (but tedious) to prove by calculations that $p_{m}^{(t)}[0, \ldots, 0]=\left((t-1) ! ! / m^{\frac{t}{2}}\right)(1-o(1))$ for even $t \leq 10$. This proves the first case in the lemma.

For the remaining two cases, we shall use generating function and some technique inspired by Fourier analysis. Consider the generating function

$$
\left(x_{1}+\ldots+x_{m}\right)^{t}=\sum_{n_{1}+\ldots+n_{m}=t}\left(\begin{array}{c}
t \\
n_{1}, \ldots, n_{m}
\end{array}\right) x_{1}^{n_{1}} \ldots x_{m}^{n_{m}} .
$$

If $x_{i} \in\{-1,1\}$, then $\left(x_{1}+\ldots+x_{m}\right)^{t}=\sum_{n_{1}+\ldots+n_{m}=t}\left(\begin{array}{c}t \\ n_{1}, \ldots, n_{m}\end{array}\right)(-1)^{\left|\left\{i: x_{i}=-1, n_{i} \equiv 1\right\}\right|}$. We sum it over all $x_{1} \ldots x_{m} \in\{-1,1\}^{m}$. Note that for those $\left(n_{1}, \ldots, n_{m}\right)$ that have some $n_{i_{0}} \equiv 1$, it holds due to the cancelation that $\sum_{x_{1}, \ldots, x_{m} \in\{-1,1\}}(-1)^{\left|\left\{i: x_{i}=-1, n_{i} \equiv 1\right\}\right|}=0$. On the other hand, if all $n_{i}$ 's are even, then $\sum_{x_{1}, \ldots, x_{m} \in\{-1,1\}}(-1)^{\left|\left\{i: x_{i}=-1, n_{i} \equiv 1\right\}\right|}=2^{m}$. Thus we have

$$
\sum_{x_{1}, \ldots, x_{m} \in\{-1,1\}}\left(x_{1}+\ldots+x_{m}\right)^{t}=2^{m} \sum_{\substack{n_{1}+\ldots+n_{m}=t \\
n_{i} \equiv 0, i \in[m]}}\left(\begin{array}{c}
t \\
n_{1}, \ldots, n_{m}
\end{array}\right) .
$$

And therefore,

$$
\begin{aligned}
p^{(t)}[0, \ldots, 0] & =\frac{1}{m^{t}} \sum_{\substack{n_{1}+\ldots+n_{m}=t \\
n_{i}=0, i \in[m]}}\left(\begin{array}{c}
t \\
n_{1}, \ldots, n_{m}
\end{array}\right) \\
& =\frac{1}{2^{m} m^{t}} \sum_{\substack{x_{1}, \ldots, x_{m} \in\{-1,1\}\\
}}\left(x_{1}+\ldots+x_{m}\right)^{t} \\
& =\frac{1}{2^{m} m^{t}} \sum_{i=0}^{m}\left(\begin{array}{c}
m \\
i
\end{array}\right)(m-2 i)^{t} \\
& =\frac{1}{2^{m}} \sum_{i=0}^{m}\left(\begin{array}{c}
m \\
i
\end{array}\right)\left(1-\frac{2 i}{m}\right)^{t} .
\end{aligned}
$$

Note that $t$ is even, so $p^{(t)}[0, \ldots, 0]$ decreases if $t$ increases by 2 , and this proves the second case of the lemma with the help of the first case. And if $t>m^{2} / 2$, then

$$
p^{(t)}[0, \ldots, 0] \leq \frac{1}{2^{m}}\left(2+\left(1-\frac{2}{m}\right)^{t} \sum_{i=1}^{m-1}\left(\begin{array}{c}
m \\
i
\end{array}\right)\right)<2 / 2^{m}+e^{-m}=O\left(1 / 2^{m}\right)
$$

This proves the third case of the lemma.

Now it is very easy to prove Theorem 2 using this lemma. For the randomized lower bound, let $m=\left\lfloor\left(n+\log _{2} n\right) / 2\right\rfloor$, then $T=\Theta\left(2^{n / 2} / n^{1 / 2}\right)$ and $\sum_{t=1}^{T} p_{t}=O(1 / n)$. Thus $R L S\left(\{0,1\}^{n}\right)=$ $\Omega\left(\sqrt{n} 2^{n / 2}\right)$. For the quantum lower bound, let $m=\left\lfloor\left(2 n+\log _{2} n\right) / 3\right\rfloor$, then $T=\Theta\left(2^{n / 3} / n^{1 / 3}\right)$ and $\sum_{t=1}^{T} \sqrt{p_{t}}=O(1 / \sqrt{n})$. Thus $Q L S\left(\{0,1\}^{n}\right)=\Omega\left(2^{n / 3} n^{1 / 6}\right)$.

\subsection{Lower bounds for Local Search on the constant dimensional grid}

In this section we shall first prove a lower bound weaker than Theorem 3 in Section 4.2.1, and then improve it to Theorem 3 in Section 4.2.2 and Section 4.2.3.

\subsubsection{A weaker family of lower bounds}

To simplify notations, we let $n=N^{1 / d}$. As in Section 4.1, we decompose the grid into two parts, $[n]^{m}$ and $[n]^{d-m}$. For each vertex $x=x_{0} \ldots x_{m-1} \in[n]^{m}$ and each $i \in\{0, \ldots, m-1\}$, define

$$
\begin{aligned}
x^{(i),-} & =x_{0} \ldots x_{i-1} \max \left\{x_{i}-1,1\right\} x_{i+1} \ldots x_{m-1}, \\
x^{(i),+} & =x_{0} \ldots x_{i-1} \min \left\{x_{i}+1, n\right\} x_{i+1} \ldots x_{m-1} .
\end{aligned}
$$


We perform the random walk $\left([n]^{m}, v_{0}^{w}, W\right)$ where $v_{0}^{w}=00 \ldots 0 \in[n]^{m}$ and

$$
W(x, t)=\left\{x^{((t-1) \bmod m),+}, x^{((t-1) \bmod m),-}\right\} .
$$

To analyze the probability $p_{t}$ in Theorem 9 , we first consider the following simpler "line walk". Suppose a particle is initially put at point $i \in\{1, \ldots, n\}$, and in each step the particle moves either to $\max \{1, i-1\}$ or to $\min \{n, i+1\}$, each with probability $1 / 2$. Let $p_{i j}^{(t)}$ denote the probability that the particle starting from point $i$ stops at point $j$ after exact $t$ steps of the walk. For $t \geq 1$, the following proposition gives a very good (actually tight) estimate on $\max _{i j} p_{i j}^{(t)}$.

Proposition 11 For any $t \geq 1$,

$$
\max _{i, j} p_{i j}^{(t)}= \begin{cases}O(1 / \sqrt{t}) & \text { if } t \leq n^{2} \\ O(1 / n) & \text { if } t>n^{2}\end{cases}
$$

Before the formal proof, let us briefly discuss the main difficulty and the idea to get around it. First note that since we care about the whole mixing process (i.e. before and after mixing), the standard eigenvalue gap does not immediately apply. Second, if there are not the two barriers (1 and $n$ ) then $p_{i j}^{(t)}$ is very easy to calculate: $p_{i j}^{(t)}=\left(\begin{array}{c}t \\ t / 2+(j-i) / 2\end{array}\right)$ if $j-i$ and $t$ have the same parity, and 0 otherwise. However, since we now have the two barriers, it is hard to count the number of paths from $i$ to $j$ after exactly $t$ steps. Fortunately, there is a basic reflecting rule as follows.

reflecting rule: In the line walk without barrier, the number of paths from $i>0$ to $j>0$ in exactly $t$ steps touching or crossing the point 0 is equal to the number of paths from $-i$ to $j$ in exactly $t$ steps.

The proof of this rule is very easy. Suppose a random path touches the point 0 at $t$ for the first time, then do a reflection of the first $t$ steps of the path with respect to point 0 . See Figure 1 for an illustration. It is not hard to see that this gives a 1-1 correspondence between the following two sets: 1) the set of paths from $i$ to $j$ after exactly $t$ steps touching or crossing the point 0 , and 2 ) the set of paths from $-i$ to $j$.

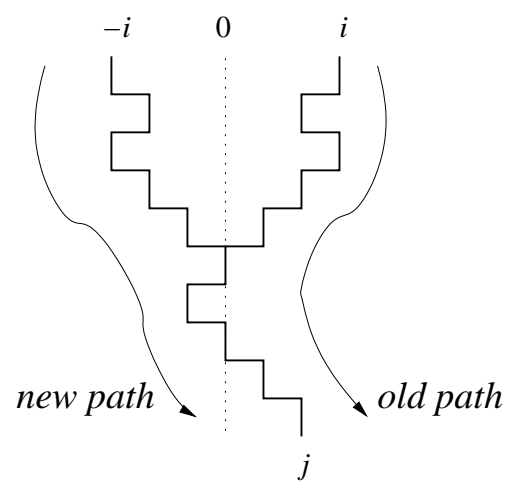

Figure 1: The proof of the reflecting rule.

Now let us consider the barrier setting. Note that a path may try to cross the two barriers in some pattern, for example, try to cross the left barrier (i.e. point 1) a times and then try to cross the right barrier (i.e. point n) $b$ times. Imagine that we now remove the two barriers, then the path will touch (from right) but not cross the point $1-a$ and will touch (from left) but not cross the point $n+b-a$. To use the reflecting rule, we just need to further note the following simple fact:

\{paths touching but not crossing the point $1-a\}$

$=\{$ paths touching or crossing the point $1-a\}-\{$ paths touching or crossing the point $-a\}$.

Following this idea, we will construct a series of 1-1 correspondences to reduce the problem step by step to the no-barrier case. The precise proof is as follows. 
Proof We consider two settings. One is the line walk on $n$ points $0, \ldots, n-1$ with the two barriers 0 and $n-1^{6}$. Another is the same except that the barriers are removed, and we have infinite points in a line. For each $t$-bit binary string $x=x_{1} \ldots x_{t}$, we use $P_{i}^{x}$ and $Q_{i}^{x}$ to denote the two paths that starting at $i$ and walk according to $x$ in the two settings. Precisely, at step $s, Q_{i}^{x}$ goes left if $x_{s}=0$ and goes right if $x_{s}=1 . P_{i}^{x}$ goes in the same way except that it will stand still if the point is currently at left (or right) end and it still wants to go left (or right). If the end point of $P_{i}^{x}$ is $j$, then we write $i \rightarrow_{t}^{P, x} j$. Let $X_{i j}^{(t), P}$ be the set of $x \in\{0,1\}^{t}$ s.t. $i \rightarrow_{t}^{P, x} j$, and put $n_{i j}^{(t), P}=\left|X_{i j}^{(t), P}\right|$. Then by definition, $p_{i j}^{(t)}=n_{i j}^{(t), P} / 2^{t}$. The notations $i \rightarrow_{t}^{Q, x} j, X_{i j}^{(t), Q}$ and $n_{i j}^{(t), Q}$ are similarly defined, with the corresponding $P$ changed to $Q$. Note that $n_{i j}^{(t), Q}=\left(\begin{array}{c}t \\ t / 2+(j-i) / 2\end{array}\right)$ if $j-i$ and $t$ have the same parity, and 0 otherwise. We now want to upper bound $n_{i j}^{(t), P}$ in terms of $n_{i j}^{(t), Q}$.

For a path $P_{i}^{x}$, if at some step it is at point 0 and wants to go left, we say it attempts to pass the left barrier. Similarly for the right barrier. We say a path is in the $\left\{a_{s}, b_{s}\right\}_{s=1}^{l}$ category if it first attempts to pass the left barrier for $a_{1}$ times, and then attempts to pass the right barrier for $b_{1}$ times, and so on. We call each round a stage $s$, which begins at the time that $P_{i}^{x}$ attempts to pass the left barrier for the $\left(a_{1}+\ldots+a_{s-1}+1\right)$-th time, and ends right before the time that $P_{i}^{x}$ attempts to pass the left barrier for the $\left(a_{1}+\ldots+a_{s}+1\right)$-th time. We also split each stage $s$ into two halves, cutting at the time right before the path attempts to pass the right barrier for the $\left(b_{1}+\ldots+b_{s-1}+1\right)$-th time. Note that $a_{1}$ may be 0 , which means that the path first attempts to pass the right barrier. Also $b_{l}$ may be 0 , which means the the last barrier the path attempts to pass is the left one. But all other $a_{i}, b_{i}$ 's are positive. Also note that in the case of $l=0$, the path never attempts to pass either barrier. Now for any fixed $l>0$, we consider those categories with $a_{1}>0$ and $b_{l}>0$. Other cases can handled similarly. Partition $X_{i j}^{(t), P}$ as

$$
X_{i j}^{(t), P}=\bigcup_{l,\left\{a_{s}, b_{s}\right\}_{s=1}^{l}} X_{i j}^{(t), P}\left[\left\{a_{s}, b_{s}\right\}_{s=1}^{l}\right]
$$

where $X_{i j}^{(t), P}\left[\left\{a_{s}, b_{s}\right\}_{s=1}^{l}\right]$ contains those $x \in\{0,1\}^{t}$ s.t. $P_{i}^{x}$ is in the category $\left\{a_{s}, b_{s}\right\}_{s=1}^{l}$. Put $n_{i j}^{(t), P}\left[\left\{a_{s}, b_{s}\right\}_{s=1}^{l}\right]=\left|X_{i j}^{(t), P}\left[\left\{a_{s}, b_{s}\right\}_{s=1}^{l}\right]\right|$, thus $n_{i j}^{(t), P}=\sum_{l} \sum_{\left\{a_{s}, b_{s}\right\}_{s=1}^{l}} n_{i j}^{(t), P}\left[\left\{a_{s}, b_{s}\right\}_{s=1}^{l}\right]$.

Now consider the corresponding paths in $X_{i j}^{(t), Q}$. The following observation relates $P_{i}^{x}$ and $Q_{i}^{x}$.

Observation 1 For each $x \in X_{i j}^{(t), P}\left[\left\{a_{s}, b_{s}\right\}_{s=1}^{l}\right]$, the following three properties hold for any $s$.

1. In the first half of stage $s$, the path $Q_{i}^{x}$ touches (from right) but does not cross the point $\alpha_{s}=\sum_{r=1}^{s-1}\left(b_{r}-a_{r}\right)-a_{s}$.

2. In the second half of stage $s$, the path $Q_{i}^{x}$ touches (from left) but does not cross the point $\beta_{s}=n-1+\sum_{r=1}^{s}\left(b_{r}-a_{r}\right)$.

3. The path $Q_{i}^{x}$ ends at $\gamma=j+\sum_{s=1}^{l}\left(b_{s}-a_{s}\right)$.

We let $Y_{i \gamma}^{(t), Q}\left[\left\{\alpha_{s}, \beta_{s}\right\}_{s=1}^{l}\right]$ contain those $x \in\{0,1\}^{t}$ satisfying the three conditions in the above observation, and denote by $m_{i \gamma}^{(t), Q}\left[\left\{\alpha_{s}, \beta_{s}\right\}_{s=1}^{l}\right]$ the size of the set $Y_{i \gamma}^{(t), Q}\left[\left\{\alpha_{s}, \beta_{s}\right\}_{s=1}^{l}\right]$. Thus the observation says $X_{i j}^{(t), P}\left[\left\{\alpha_{s}, \beta_{s}\right\}_{s=1}^{l}\right] \subseteq Y_{i j}^{(t), Q}\left[\left\{\alpha_{s}, \beta_{s}\right\}_{s=1}^{l}\right]$, and therefore we have $n_{i j}^{(t), P}\left[\left\{a_{s}, b_{s}\right\}_{s=1}^{l}\right] \leq$ $m_{i \gamma}^{(t), Q}\left[\left\{\alpha_{s}, \beta_{s}\right\}_{s=1}^{l}\right]$. So it is enough to upper bound $m_{i \gamma}^{(t), Q}\left[\left\{\alpha_{s}, \beta_{s}\right\}_{s=1}^{l}\right]$.

Now for each $x \in Y_{i \gamma}^{(t), Q}\left[\left\{\alpha_{s}, \beta_{s}\right\}_{s=1}^{l}\right]$, if we change the condition 1 in state $s=1$ by allowing the path to cross the point $\alpha_{1}$, and let $Z_{i \gamma}^{(t), Q}\left[\left\{\alpha_{s}, \beta_{s}\right\}_{s=1}^{l}\right]$ be the new set satisfying the new conditions, then $m_{i \gamma}^{(t), Q}\left[\left\{\alpha_{s}, \beta_{s}\right\}_{s=1}^{l}\right]=\left|Z_{i \gamma}^{(t), Q}\left[\left\{\alpha_{s}, \beta_{s}\right\}_{s=1}^{l}\right]\right|-\left|Z_{i \gamma}^{(t), Q}\left[\alpha_{1}-1, \beta_{1},\left\{\alpha_{s}, \beta_{s}\right\}_{s=2}^{l}\right]\right|$. In other words, the set of paths touches (from right) but does not cross $\alpha_{1}$ is the set of paths touches or crosses $\alpha_{1}$ minus the set of paths touches or crosses $\alpha_{1}-1$.

Now we calculate $\left|Z_{i \gamma}^{(t), Q}\left[\left\{\alpha_{s}, \beta_{s}\right\}_{s=1}^{l}\right]\right|$ by the so-called reflection rule. Suppose the first time that $Q_{i}^{x}$ touches $\alpha_{1}$ is $t_{1}$. We reflect the first $t_{1}$ part of the path $Q_{i}^{x}$ with respect to the point $\alpha_{1}$. Precisely,

\footnotetext{
${ }^{6}$ Here we let the $n$ points be $0, \ldots, n-1$ instead of $1, \ldots, n$ just to make the later calculation cleaner.
} 
let $y=\left(1-x_{1}\right) \ldots\left(1-x_{t_{1}}\right) x_{t_{1}+1} \ldots x_{t}$, then the paths $Q_{i}^{x}$ and $Q_{2 \alpha_{1}-i}^{y}$ merge at time $t_{1}$. And it is easy to check that it is a 1-1 correspondence between $Z_{i \gamma}^{(t), Q}\left[\left\{\alpha_{s}, \beta_{s}\right\}_{s=1}^{l}\right]$ and $Y_{2 \alpha_{1}-i, \gamma}^{(t) Q}\left[\beta_{1},\left\{\alpha_{s}, \beta_{s}\right\}_{s=2}^{l}\right]$, Here $Y_{2 \alpha_{1}-i, \gamma}^{(t), Q}\left[\beta_{1},\left\{\alpha_{s}, \beta_{s}\right\}_{s=2}^{l}\right]$ is the set of paths starting at $2 \alpha_{1}-i$, satisfying (a) the condition 2 at the first stage, (b) both conditions 1 and 2 at the rest $l-1$ stages, and (c) condition 3 . So

$$
\begin{aligned}
\left|Z_{i \gamma}^{(t), Q}\left[\left\{\alpha_{s}, \beta_{s}\right\}_{s=1}^{l}\right]\right| & =\left|Y_{2 \alpha_{1}-i, \gamma}^{(t), Q}\left[\beta_{1},\left\{\alpha_{s}, \beta_{s}\right\}_{s=2}^{l}\right]\right|=m_{2 \alpha_{1}-i, \gamma}^{(t), Q}\left[\beta_{1},\left\{\alpha_{s}, \beta_{s}\right\}_{s=2}^{l}\right] \\
& =m_{-2 a_{1}-i, \gamma}^{(t), Q}\left[\beta_{1},\left\{\alpha_{s}, \beta_{s}\right\}_{s=2}^{l}\right] \\
& =m_{-a_{1}-i, \gamma+a_{1}}^{(t), Q}\left[\beta_{1}+a_{1},\left\{\alpha_{s}+a_{1}, \beta_{s}+a_{1}\right\}_{s=2}^{l}\right]
\end{aligned}
$$

where (70) is due to the fact that $\alpha_{1}=-a_{1}$, and (71) is because that the number of the paths does not change if we move all the paths right by $a_{1}$. Similarly, we have

$$
\begin{aligned}
\left|Z_{i \gamma}^{(t), Q}\left[\alpha_{1}-1, \beta_{1},\left\{\alpha_{s}, \beta_{s}\right\}_{s=2}^{l}\right]\right| & =m_{2 \alpha_{1}-2-i, \gamma}^{(t), Q}\left[\beta_{1},\left\{\alpha_{s}, \beta_{s}\right\}_{s=2}^{l}\right] \\
& =m_{-a_{1}-2-i, \gamma+a_{1}}^{(t), Q}\left[\beta_{1}+a_{1},\left\{\alpha_{s}+a_{1}, \beta_{s}+a_{1}\right\}_{s=2}^{l}\right]
\end{aligned}
$$

Therefore,

$$
\begin{aligned}
n_{i j}^{(t), P}\left[\left\{a_{s}, b_{s}\right\}_{s=1}^{l}\right] \leq & m_{i \gamma}^{(t), Q}\left[\left\{\alpha_{s}, \beta_{s}\right\}_{s=1}^{l}\right] \\
= & m_{-2 a_{1}-i, \gamma}^{(t), Q}\left[\beta_{1},\left\{\alpha_{s}, \beta_{s}\right\}_{s=2}^{l}\right]-m_{-2 a_{1}-2-i, \gamma}^{(t), Q}\left[\beta_{1},\left\{\alpha_{s}, \beta_{s}\right\}_{s=2}^{l}\right] \\
= & m_{-a_{1}-i, \gamma+a_{1}}^{(t), Q}\left[\beta_{1}+a_{1},\left\{\alpha_{s}+a_{1}, \beta_{s}+a_{1}\right\}_{s=2}^{l}\right] \\
& -m_{-a_{1}-2-i, \gamma+a_{1}}^{(t),}\left[\beta_{1}+a_{1},\left\{\alpha_{s}+a_{1}, \beta_{s}+a_{1}\right\}_{s=2}^{l}\right]
\end{aligned}
$$

Note that $\alpha_{s}+a_{1}=b_{1}+\sum_{r=2}^{s-1}\left(b_{r}-a_{r}\right)-a_{s}, \quad \beta_{s}+a_{1}=n-1+b_{1}+\sum_{r=2}^{s}\left(b_{r}-a_{r}\right)$ and $\gamma+a_{1}=j+b_{1}+\sum_{r=2}^{s}\left(b_{r}-a_{r}\right)$ are all functions of $\left(b_{1}, a_{2}, b_{2}, \ldots, a_{l}, b_{l}\right)$, not of $a_{1}$ any more. Therefore,

$$
\begin{aligned}
& \sum_{a_{1}, b_{1}, \ldots, a_{l}, b_{l}>0} n_{i j}^{(t), P}\left[\left\{a_{s}, b_{s}\right\}_{s=1}^{l}\right] \\
\leq & \sum_{b_{1}, \ldots, a_{l}, b_{l}>0} \sum_{a_{1}>0}\left(m_{-a_{1}-i, \gamma+a_{1}}^{(t), Q}\left[\beta_{1}+a_{1},\left\{\alpha_{s}+a_{1}, \beta_{s}+a_{1}\right\}_{s=2}^{l}\right]\right. \\
& \quad \sum_{b_{1}, \ldots, a_{l}, b_{l}>0}^{(t), Q}\left(m_{-1-i, \gamma+a_{1}}^{(t), Q}\left[\beta_{1}+a_{1},\left\{\alpha_{s}+a_{1}, \beta_{s}+a_{1}\right\}_{s=2}^{l}\right]\right. \\
& \left.\quad \sum_{b_{1}, \ldots, a_{l}, b_{l}>0} 2 m_{h=1,2}^{(t), Q} \max _{-2-i, \gamma+a_{1}}\left[\beta_{1}+a_{1},\left\{\alpha_{s}+a_{1}, \beta_{s}+a_{1}\right\}_{s=2}^{l}\right]\right)
\end{aligned}
$$

Now using the similar methods, i.e. reflecting with respect to points $\left(n-1+b_{1}\right)$ and $\left(n+b_{1}\right)$, moving the paths left by $b_{1}$, and finally collapsing the telescope, we can get

$$
\begin{aligned}
& \sum_{b_{1}, \ldots, a_{l}, b_{l}>0} m_{-h-i, \gamma+a_{1}}^{(t), Q}\left[\beta_{1}+a_{1},\left\{\alpha_{s}+a_{1}, \beta_{s}+a_{1}\right\}_{s=2}^{l}\right] \\
\leq & \sum_{a_{2}, b_{2}, \ldots, a_{l}, b_{l}>0} 2 \max _{k=1,2}\left\{m_{2 n+i+h-k+1, \gamma+a_{1}-b_{1}}^{(t), Q}\left[\left\{\alpha_{s}+a_{1}-b_{1}, \beta_{s}+a_{1}-b_{1}\right\}_{s=2}^{l}\right]\right\}
\end{aligned}
$$

and thus

$$
\begin{aligned}
& \sum_{a_{1}, b_{1}, \ldots, a_{l}, b_{l}>0} n_{i j}^{(t), P}\left[\left\{a_{s}, b_{s}\right\}_{s=1}^{l}\right] \\
\leq & \sum_{a_{2}, b_{2}, \ldots, a_{l}, b_{l}>0} 4 \max _{h=0,1,2}\left\{m_{2 n+i+h, \gamma+a_{1}-b_{1}}^{(t), Q}\left[\left\{\alpha_{s}+a_{1}-b_{1}, \beta_{s}+a_{1}-b_{1}\right\}_{s=2}^{l}\right]\right\}
\end{aligned}
$$


We continue this process, and finally we get

$$
\begin{aligned}
\sum_{a_{1}, b_{1}, \ldots, a_{l}, b_{l}>0} n_{i j}^{(t), P}\left[\left\{a_{s}, b_{s}\right\}_{s=1}^{l}\right] & \leq 2^{2 l} \max _{h=0,1, \ldots, 2 l} n_{2 l n+i+h, \gamma+\sum_{s=1}^{l}\left(a_{s}-b_{s}\right)}^{(t),} \\
& =2^{2 l} \max _{h=0,1, \ldots, 2 l} n_{2 l n+i+h, j}^{(t), Q} \\
& =2^{2 l} n_{2 l, Q+i, j}^{(t), Q} \\
& \leq 2^{2 l}\left(\begin{array}{c}
t \\
\frac{t}{2}+\frac{j-i-2 l n}{2}
\end{array}\right)
\end{aligned}
$$

Thus

$$
\begin{aligned}
& \sum_{l>0} \sum_{a_{1}, b_{1}, \ldots, a_{l}, b_{l}>0} n_{i j}^{(t), P}\left[\left\{a_{s}, b_{s}\right\}_{s=1}^{l}\right] \\
\leq & \sum_{l \geq 0} 2^{2(l+1)}\left(\begin{array}{c}
t \\
\frac{t}{2}+l n
\end{array}\right) \\
= & 4\left(\begin{array}{c}
t \\
t / 2
\end{array}\right)+\sum_{l \geq 1} 2^{2(l+1)}\left(\begin{array}{c}
t \\
t / 2+l n
\end{array}\right) \\
\leq & 4\left(\begin{array}{c}
t \\
t / 2
\end{array}\right)+\frac{1}{n} \sum_{l \geq 1} 2^{2(l+1)}\left(\left(\begin{array}{c}
t \\
t / 2+l n
\end{array}\right)+\left(\begin{array}{c}
t \\
t / 2+l n-1
\end{array}\right)+\ldots+\left(\begin{array}{c}
t \\
t / 2+l n-n+1
\end{array}\right)\right) \\
\leq & 4\left(\begin{array}{c}
t \\
t / 2
\end{array}\right)+\frac{1}{n} \sum_{l \geq 1} 2^{2(l+1)}\left(\left(\begin{array}{c}
t \\
t
\end{array}\right)+\left(\begin{array}{c}
t \\
t-1
\end{array}\right)+\ldots+\left(\begin{array}{c}
t \\
t / 2+l n-n+1
\end{array}\right)\right) \\
\leq & 4\left(\begin{array}{c}
t \\
t / 2
\end{array}\right)+\frac{1}{n} \sum_{l \geq 1} 2^{2(l+1)} 2^{t} e^{-\frac{2(l-1)^{2} n^{2}}{3 t}}
\end{aligned}
$$

where $\left(\begin{array}{c}t \\ t^{\prime}\end{array}\right)=0$ if $t^{\prime}>t$. Here the first two inequalities are by the monotonicity of binomial coefficients, and the last inequality is by Chernoff's Bound. Now if $t \leq n^{2}$, then $\sum_{l \geq 1} 2^{2(l+1)} e^{-\frac{2(l-1)^{2} n^{2}}{3 t}} \leq$ $\sum_{l \geq 1} 2^{2(l+1)} e^{-\frac{2(l-1)^{2}}{3}}=O(1)$, so $\sum_{l>0} \sum_{a_{1}, b_{1}, \ldots, a_{l}, b_{l}>0} n_{i j}^{(t), P}\left[\left\{a_{s}, b_{s}\right\}_{s=1}^{l}\right] \leq O\left(\left(\begin{array}{c}t \\ t / 2\end{array}\right)+2^{t} / n\right)=$ $O\left(2^{t} / \sqrt{t}\right)$. For other categories of $a_{1}=0$ or $b_{l}=0$, the same result can be proved similarly, and the $l=0$ is easy since $n_{i j}^{(t), Q}=O\left(2^{t} / \sqrt{t}\right)$. Putting all things together, we see that $p_{i j}^{(t)}=O(1 / \sqrt{t})$ if $t \leq n^{2}$. The other part, i.e. $p_{i j}^{(t)}=O(1 / n)$ when $t>n^{2}$, can be easily derived from this and the fact that $\max _{i j} p_{i j}^{(t)}$ decreases as $t$ increases. This completes our proof.

Now we use Proposition 11 to prove the weaker lower bounds for grids. Note that the random walk $\left([n]^{m}, v_{0}^{w}, W\right)$ is just a product of $m$ line walks, i.e. cyclicly perform the line walk in the cyclic order of dimension $0,1, \ldots, m-1$ (see Eq. (66)). Therefore, the $p_{t}$ in the random walk $\left([n]^{m}, v_{0}^{w}, W\right)$ satisfies

$$
p_{t}= \begin{cases}O\left(1 / \sqrt{t^{m}}\right) & \text { if } t \leq n^{2} \\ O\left(1 / n^{m}\right) & \text { if } t>n^{2}\end{cases}
$$

Now for the randomized lower bounds, when $d>4$ we pick $m=\lceil d / 2\rceil>2$ and we get

$$
R L S\left([n]^{d}\right)=\Omega\left(\frac{n^{d-m}}{O(1)+n^{d-m} / n^{m}}\right)=\Omega\left(n^{\lfloor d / 2\rfloor}\right)= \begin{cases}\Omega\left(n^{\frac{d}{2}}\right) & \text { if } d \text { is odd }, \\ \Omega\left(n^{\frac{d}{2}-\frac{1}{2}}\right) & \text { if } d \text { is even. }\end{cases}
$$

For $d=4,3,2$, we let $m=2,2,1$ respectively, and get $R L S\left([n]^{4}\right)=\Omega\left(n^{2} /(\log n+1)\right)=\Omega\left(n^{2} / \log n\right)$, $R L S\left([n]^{3}\right)=\Omega(n /(\log n+1 / n))=\Omega(n / \log n)$, and $R L S\left([n]^{2}\right)=\Omega(n /(\sqrt{n}+1))=\Omega(\sqrt{n})$. 
For the quantum lower bounds, if $d>6$, we let $m$ be the integer closest to $2 d / 3$, thus $m>4$. We get

$$
Q L S\left([n]^{d}\right)=\Omega\left(\frac{n^{d-m}}{O(1)+n^{d-m} / n^{m / 2}}\right)=\left\{\begin{array}{ll}
\Omega\left(N^{\frac{1}{3}}\right) & \text { if } d=3 d^{\prime} \\
\Omega\left(N^{\frac{1}{3}-\frac{1}{3 d}}\right) & \text { if } d=3 d^{\prime}+1 . \\
\Omega\left(N^{\frac{1}{3}-\frac{1}{6 d}}\right) & \text { if } d=3 d^{\prime}+2
\end{array} .\right.
$$

For $d=6$, let $m=4$ and we have $Q L S\left([n]^{6}\right)=\Omega\left(n^{2} / \log n\right)$. For $d=5,4,3$, we let $m=d-2$ and then $Q L S\left([n]^{d}\right)=\Omega\left(n^{2} /\left(n^{2-(d-2) / 2}+n^{2-(d-2) / 2}\right)\right)=\Omega\left(n^{d / 2-1}\right)$, which is $\Omega\left(n^{5 / 2}\right), \Omega\left(n^{2}\right), \Omega\left(n^{3 / 2}\right)$, respectively. For $d=2$, let $m=1$ and $Q L S\left([n]^{2}\right)=\Omega\left(\frac{n}{n^{3 / 4}}\right)=\Omega\left(n^{1 / 4}\right)$.

\subsubsection{Improvement}

One weakness of the above proof is the integer constraint of the dimension $m$. We now show a way to get around the problem, allowing $m$ to be any real number between 0 and $d-1$. The idea is to partition the grid into many blocks, with different blocks representing different time slots, and the blocks are threaded into one very long block by many paths that are pairwise disjoint. Roughly speaking, we view $[n]^{d}$ as the product of $d$ line graph $[n]$. For each of the first $d-1$ line graphs, we cut it into $n^{1-r}$ parts evenly, each of size $n^{r}$. (Here $r=m /(d-1)$ ). Then $[n]^{d-1}$ is partitioned into $n^{(d-1)(1-r)}$ smaller grids, all isomorphic to $\left[n^{r}\right]^{d-1}$. Putting the last dimension back, we have $n^{(d-1)(1-r)}$ blocks, all isomorphic to $\left[n^{r}\right]^{d-1} \times[n]$. Now the random walk will begin in the first block, and within each block, there is just one step of random walk in $\left[n^{r}\right]^{d-1}$ followed by two steps of one-way walk in the last dimension space $[n]$. When the walk runs out of the clock $[n]$, the walk will move to the next block via a particular block-changing path. All block-changing paths are carefully designed to be disjoint, and they "thread" all the blocks to form a $\left[n^{r}\right]^{d-1} \times[L]$ grid, where $L=\left(n-2 n^{r}\right) n^{(1-r)(d-1)}$. ( $L$ is not $n \cdot n^{(1-r)(d-1)}$ because we need $2 n^{r}$ points for the block-changing paths.) Figure 2 is an illustration for the case of $d=2$.

We now describe the partition and the walk precisely. For $x=x_{0} \ldots x_{d-1}$ in $[n]^{d}$, let $x^{(k)=l}=$ $x_{0} \ldots x_{k-1} l x_{k+1} \ldots x_{d-1}$, and $x^{(k)=(k)+i}=x_{0} \ldots x_{k-1}\left(x_{k}+i\right) x_{k+1} \ldots x_{d-1}$, where $i$ satisfies $x_{k}+i \in[n]$. Recall that $x^{(i),-}=x^{(i)=\max \left\{x_{i}-1,1\right\}}$ and $x^{(i),+}=x^{(i)=\min \left\{x_{i}+1, n\right\}}$.

For any fixed constant $r \in(0,1)$, let $\alpha=\left\lfloor n^{r}\right\rfloor, \beta=\left\lfloor n^{1-r}\right\rfloor$ and $n^{\prime}=\alpha \beta$. Note that $n^{\prime} \geq$ $\left(n^{r}-1\right)\left(n^{1-r}-1\right)=n-o(n)$. We now consider the slightly smaller grid $\left[n^{\prime}\right]^{d}$. Let $V_{1}$ be the set $\left[n^{\prime}\right]^{d-1}=\left\{x_{0} \ldots x_{d-2}: x_{i} \in\left[n^{\prime}\right]\right\}$. We cut $V_{1}$ into $\beta^{d-1}$ parts $\left\{x_{0} \ldots x_{d-2}:\left(k_{i}-1\right) \alpha<x_{i} \leq\right.$ $\left.k_{i} \alpha\right\}_{k_{0} \ldots k_{d-2} \in[\beta]^{d-1}}$, each of which is a small grid isomorphic to $[\alpha]^{d-1}$. We then refer to the set $\left\{x_{0} \ldots x_{d-2} x_{d-1}:\left(k_{i}-1\right) \alpha<x_{i} \leq k_{i} \alpha, i=0, \ldots, d-2, \alpha<x_{d-1} \leq n^{\prime}-\alpha\right\}$ as the "block $\left(k_{0}, \ldots, k_{d-2}\right)$ ". Note that $\left(k_{0}, \ldots, k_{d-2}\right)$ can be also viewed as a point in grid $[\beta]^{d-1}$, and there is a Hamilton path $\operatorname{HamPath}_{\beta, d-1}$ in $[\beta]^{d-1}$, as defined in Section 2. We call the block $\left(k_{0}^{\prime}, \ldots, k_{d-2}^{\prime}\right)$ the next block of the block $\left(k_{0}, \ldots, k_{d-2}\right)$ if $\left(k_{0}^{\prime}, \ldots, k_{d-2}^{\prime}\right)$, viewed as the point in $[\beta]^{d-1}$, is the next point of $\left(k_{0}, \ldots, k_{d-2}\right)$ in $\operatorname{HamPath}_{\beta, d-1}$. Note that by our definition of $\operatorname{HamPath}_{\beta, d-1}$, we know that $\exists i \in\{0, \ldots, d-2\}$ s.t. $k_{i}^{\prime} \in\left\{k_{i}+1, k_{i}-1\right\}$ and for all other $j \neq i, k_{j}^{\prime}=k_{j}$. That is, adjacent blocks have only one coordinate to be different, and this difference is 1 . We call the block $\left(k_{0}, \ldots, k_{d-2}\right)$ the last block if $\left(k_{0}, \ldots, k_{d-2}\right)$ is the last point in $\operatorname{HamPath}_{\beta, d-1}$.

Now we define the random walk by describing how a particle may go from start to end. The path set is just all the possible paths the particle goes along. Intuitively, within one block, the last dimension $d-1$ serves as the clock space. So as before, we perform one step of line walk (in the dimension which is the circularly next dimension of the last one that the walk just goes in), followed by two steps of walk in the clock space. If we run out of clock, we say we reach a boundary point at the current block, and we move to the next block via a path segment called block-changing segment. In what follows, we specify how the particle may move during the whole random walk process, including going through block-changing segments. We always use $x_{0} \ldots x_{d-1}$ to denote the current position of the particle, and assume $x_{i}=\left(k_{i}-1\right) \alpha+y_{i}$, i.e. $x$ is in the block $\left(k_{0}, \ldots, k_{d-2}\right)$ with the offsets $\left(y_{0}, \ldots, y_{d-1}\right)$. Thus the instruction $x_{0}=x_{0}+1$, for example, means that the particle moves from $x_{0} \ldots x_{d-1}$ to $\left(x_{0}+1\right) x_{1} \ldots x_{d-1}$.

1. Initially $x_{0}=\ldots=x_{d-2}=0, x_{d-1}=\alpha+1, k_{0}=\ldots=k_{d-2}=1$. 


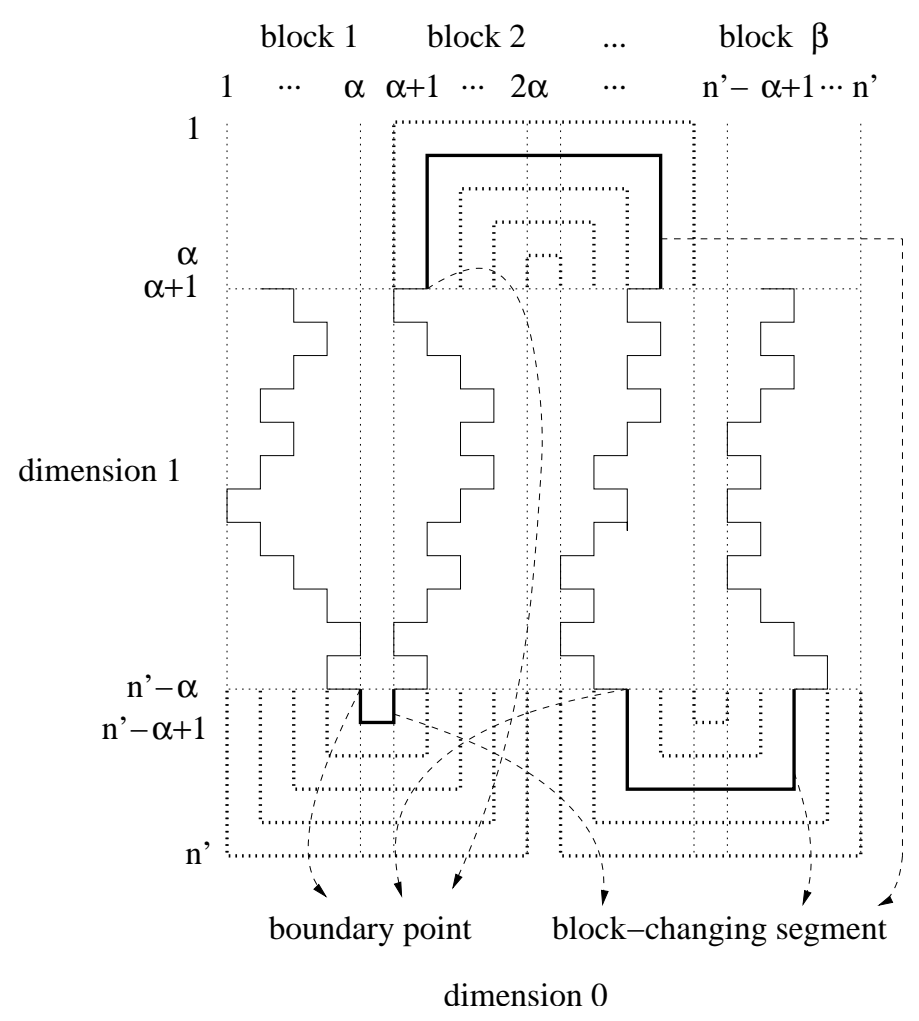

Figure 2: Illustration for changing a block in the 2-dimensional grid

2. for $t=1$ to $\left(n^{\prime}-2 \alpha\right) \beta^{d-1}$,

Let $t^{\prime}=\left\lfloor\frac{t-1}{n^{\prime}-2 \alpha}\right\rfloor, i=(t-1) \bmod (d-1)$

do either $x_{i}=\max \left\{x_{i}-1,\left(k_{i}-1\right) \alpha+1\right\}$ or $x_{i}=\min \left\{x_{i}+1, k_{i} \alpha\right\}$ randomly

if $t \neq k\left(n^{\prime}-2 \alpha\right)$ for some positive integer $k$,

do $x_{d-1}=x_{d-1}+(-1)^{t^{\prime}}$ twice

else (the particle is now at a boundary point)

if the particle is not in the last block

(Suppose the current block changes to the next block by increasing $k_{j}$ by $b \in\{-1,1\}$ )

$$
\begin{aligned}
& \text { do } x_{d-1}=x_{d-1}+(-1)^{t^{\prime}} \text { for }\left(\alpha+1-y_{j}\right) \text { times } \\
& \text { do } x_{j}=x_{j}+b \text { for } 2\left(\alpha+1-y_{j}\right)-1 \text { times } \\
& \text { do } x_{d-1}=x_{d-1}+(-1)^{t^{\prime}+1} \text { for }\left(\alpha+1-y_{j}\right) \text { times } \\
& k_{j}=k_{j}+b
\end{aligned}
$$

else

The particle stops and the random walk ends

It is easy to verify that every boundary point has one unique block-changing segment, and different block-changing segments do not intersect. Also note that we do not let the clock tick when we are moving from one block to another. Thus the block-changing segments thread all the blocks to form a $[\alpha]^{d-1} \times[L]$ grid, where $L=\left(n^{\prime}-2 \alpha\right) \beta^{d-1}$. Actually, for our lower bound purpose, we can think of the random walk as performed in the product graph $[\alpha]^{d-1} \times[L]$. We will make this clearer below.

What we care about is, as before, the probability that the random walk starting from a point $x=x_{0} \ldots x_{d-1}$ passes another point $x^{\prime}=x_{0}^{\prime} \ldots x_{d-1}^{\prime}$. Note that for any point $x$ (including those on the block-changing segments), there is only one time $t$ when the walk may hit $x$, and this $t$ is 
determined by $x$ itself. Similarly we use $t^{\prime}$ to denote the time when the path passes $x^{\prime}$. Denote the probability that the random walk starting from $x$ passes $x^{\prime}$ by $\operatorname{Pr}\left[x \rightarrow x^{\prime}\right]$. As before suppose $x_{i}=\left(k_{i}-1\right) \alpha+y_{i}$ and $x_{i}^{\prime}=\left(k_{i}^{\prime}-1\right) \alpha+y_{i}^{\prime}$ for $i \in\{0, \ldots, d-2\}$.

We first consider the case that one of the two points, say $x^{\prime}$ is on a block-changing segment. Since different block-changing segments never intersect, a path passes $x^{\prime}$ if and only if the path passes the boundary point $x^{\prime \prime}$ at the beginning of the block-changing segment that $x^{\prime}$ is in. Also note that the time that the path passes $x^{\prime \prime}$ is also $t^{\prime}$ because the time does not elapse on the block-changing segment. So we have that $\operatorname{Pr}\left[x \rightarrow x^{\prime}\right]=\operatorname{Pr}\left[x \rightarrow x^{\prime \prime}\right]$, and it is enough to consider the case that both $x$ and $x^{\prime}$ are not in clock-changing segments.

Now suppose both $x$ and $x^{\prime}$ are not in clock-changing segments. In general, $x$ and $x^{\prime}$ may be not in the same block, so going from $x$ to $x^{\prime}$ needs to change blocks. Recall that to change from the block $\left(k_{0}, \ldots, k_{d-2}\right)$ to the next one, only one $k_{i}$ changes by increasing or decreasing by 1 . Suppose that to go to $x^{\prime}$ from $x$, we change blocks for $c$ times, by changing $k_{i_{1}}, k_{i_{2}}, \ldots, k_{i_{c}}$ in turn. Let $n_{j}=\left|\left\{s \in[c]: i_{s}=j\right\}\right|$. Note that to get to $x^{\prime}$ from $x$ after $t^{\prime}-t$ steps, the coordinate $j$ needs to be $x_{j}^{\prime}$ after $t^{\prime}-t$ steps for each coordinate $j \in\{0, \ldots, d-2\}$. It is not hard to see that if a blockchanging needs to change $k_{j}$ by increasing $b \in\{-1,1\}$, then among all the offsets $y_{i}$ 's, only the $y_{j}$ gets changed, and the change is a reflection within the block. That is, suppose $x_{j}$ is $\left(k_{j}-1\right) \alpha+y_{j}$ before the block-changing, then $x_{j}$ changes to $\left(k_{j}+b-1\right) \alpha+\left(\alpha+1-y_{j}\right)$ after the block-changing. So if $c=1$, then $\operatorname{Pr}\left[x \rightarrow x^{\prime}\right]$ is equal to the probability that a random walk in $[\alpha]^{d-1}$ starting from $y_{0} \ldots y_{d-2}$ hits $y_{0}^{\prime \prime} \ldots y_{d-2}^{\prime \prime}$ after exactly $t^{\prime}-t$ steps, where $y_{j}^{\prime \prime}=y_{j}^{\prime}$ if $j \neq i_{1}$ and $y_{i_{1}}^{\prime \prime}=\alpha+1-y_{i_{1}}^{\prime}$. For general $c, \operatorname{Pr}\left[x \rightarrow x^{\prime}\right]$ is equal to the probability that a random walk in $[\alpha]^{d-1}$ starting from $y_{0} \ldots y_{d-2}$ hits $y_{0}^{\prime \prime} \ldots y_{d-2}^{\prime \prime}$ after exactly $t^{\prime}-t$ steps, where $y_{j}^{\prime \prime}=y_{j}^{\prime}$ if $n_{j}$ is even and $y_{j}^{\prime \prime}=\alpha+1-y_{j}^{\prime}$ if $n_{j}$ is odd. Note that this probability has nothing to do with the block-changing; it is just the same as we have a clock space $\left[\left(n^{\prime}-2 \alpha\right) \beta^{d-1}\right]$ to record the random walk on $[\alpha]^{d-1}$. Thus we can use Proposition 11 to upper bound this probability and just think of the graph as $\left[n^{r}\right]^{d-1} \times[L]$ and use Theorem 9, with $G^{w}=\left[n^{r}\right]^{d-1}$ and $G^{c}=[L]$.

Now we have $T=\lfloor L / 2\rfloor$ and $p_{t}=O\left(1 / \sqrt{t^{d-1}}\right)$ for $t \leq n^{2 r}$ and $p_{t}=O\left(1 / n^{r(d-1)}\right)$ for $t>n^{2 r}$. So for randomized lower bounds, if $d \geq 4$, then let $r=d /(2 d-2)$ and we get

$$
R L S\left([n]^{d}\right)=\Omega\left(n^{1+(1-r)(d-1)} /\left(\sum_{t=1}^{n^{d /(d-1)}} \frac{1}{\sqrt{t^{d-1}}}+\frac{n^{1+(1-r)(d-1)}}{n^{r(d-1)}}\right)\right)=\Omega\left(n^{d / 2}\right) .
$$

If $d=3$, let $r=3 / 4-\log \log n /(4 \log n)$, and we get $R L S\left([n]^{3}\right)=\Omega\left(\left(n^{3} / \log n\right)^{1 / 2}\right)$. For $d=2$, let $r=2 / 3$ and we get $R L S\left([n]^{2}\right)=\Omega\left(n^{2 / 3}\right)$.

For the quantum lower bounds, if $d \geq 6$, then let $r=2 d /(3 d-3)$ and we get

$$
Q L S\left([n]^{d}\right)=\Omega\left(n^{1+(1-r)(d-1)} /\left(\sum_{t=1}^{n^{d /(d-1)}} \frac{1}{t^{(d-1) / 4}}+\frac{n^{1+(1-r)(d-1)}}{n^{r(d-1) / 2}}\right)\right)=\Omega\left(n^{d / 3}\right) .
$$

If $d=5$, then let $r=5 / 6-\log \log n /(6 \log n)$ and $Q L S\left([n]^{5}\right)=\Omega\left(\left(n^{5} / \log n\right)^{1 / 3}\right)$. For $2 \leq d \leq 4$, we let $r=d /(d+1)$, then $Q L S\left([n]^{d}\right)=\Omega\left(n^{d / 2-d /(d+1)}\right)$, which is $\Omega\left(n^{1 / 3}\right), \Omega\left(n^{3 / 4}\right), \Omega\left(n^{6 / 5}\right)$ for $d=2,3,4$, respectively.

\subsubsection{Further improvement on 2-dimensional grid $[n]^{2}$}

Some other random walk may be used to further improve the lower bound on low dimension grid cases. Here is one way to improve $Q L S\left([n]^{2}\right)$ from $\Omega\left(n^{1 / 3}\right)$ to $\Omega\left(n^{4 / 5}\right)$. We cut the graph $[n]^{2}$ into $n^{2 / 5}$ smaller grids, each of size $n^{4 / 5} \times n^{4 / 5}$. Without loss of generality, assume both $n^{1 / 5}$ and $n^{4 / 5}$ are integers, and further assume $n^{1 / 5}=3 \bmod 4$; otherwise we can consider a slightly smaller grid by the simple trick as at the beginning of Section 4.2.2. We shall use a random walk similar to Aaronson's in [2] as follows in each block, and change blocks after each step. Thus different blocks record different time. 
For any time $t \in\left[n^{1 / 5}\left(n^{1 / 5}-1\right)\right]$, suppose $t=2 r n^{1 / 5}+t^{\prime}$ where $r \in\left\{0,1, \ldots,\left(n^{1 / 5}-3\right) / 2\right\}$ and $t^{\prime} \in\left\{1,2, \ldots, 2 n^{1 / 5}\right\}$. Let

$$
u= \begin{cases}0 & \text { if } t^{\prime} \equiv 0,1(\bmod 4) \\ n^{4 / 5} & \text { if } t^{\prime} \equiv 2,3(\bmod 4) .\end{cases}
$$

Let $\operatorname{block}(t)$ as the small grid

$$
\begin{cases}\left\{\left(x=\left(\left\lceil t^{\prime} / 2\right\rceil-1\right) n^{4 / 5}+x^{\prime}, y=2 r n^{4 / 5}+u+y^{\prime}\right): x^{\prime}, y^{\prime} \in\left[n^{4 / 5}\right]\right\} & \text { if } r \text { is even } \\ \left\{\left(x=\left(n^{1 / 5}-\left\lceil t^{\prime} / 2\right\rceil\right) n^{4 / 5}+x^{\prime}, y=2 r n^{4 / 5}+u+y^{\prime}\right): x^{\prime}, y^{\prime} \in\left[n^{4 / 5}\right]\right\} & \text { if } r \text { is odd }\end{cases}
$$

The $\left(x^{\prime}, y^{\prime}\right)$ is called the offset of $(x, y)$. Now define the random walk as follows and as depicted in Figure 3.

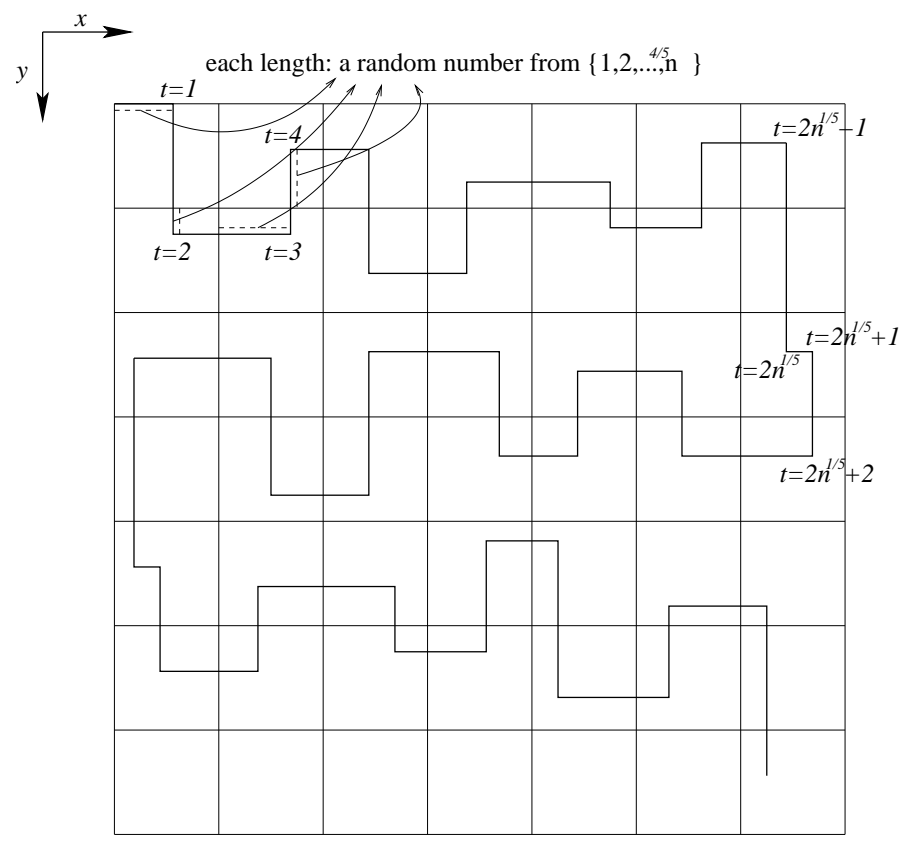

Figure 3: A different random walk in the 2 dimensional-grid

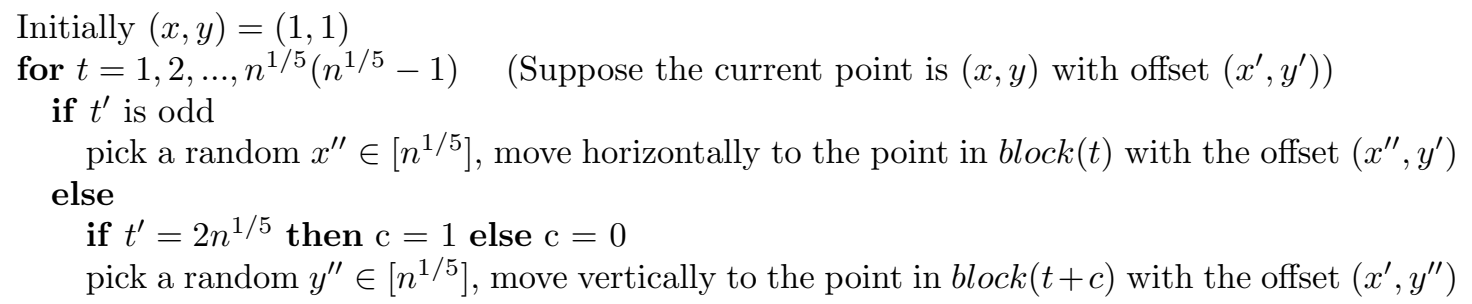

We then follow the same track as in the proof of Theorem 9. To get a reduction from Local Search on $[n]^{2}$ to the $\operatorname{Path}_{P}$ problem, we define the function

$$
f_{X}(v)= \begin{cases}l_{[n]^{2}}(v,(1,1)) & \text { if } v \notin \operatorname{set}(X) \\ -2 n^{4 / 5}(t-1)-(-1)^{r} x_{v}^{\prime}+(-1)^{\left\lceil t^{\prime} / 2\right\rceil} y_{v}^{\prime} & \text { if } v \in \operatorname{set}(X) \cap \operatorname{block}(t) .\end{cases}
$$

Intuitively, the function value decreases along the path as before. But the decrement is not always by 1: each block has its fixed value setting. If for example the path passes through the block toward 
right and down (as in the first block), then the value $-x^{\prime}-y^{\prime}$ is used within the block. In this way, we do not need to know the length of the path segment from top to $v$ to calculate each $f_{X}(v)$.

What we care about is still, as in Equality (35), the probability that the path $X^{\prime}$ passes another point $x$ on $X$, under the condition that $X^{\prime} \wedge Y=k^{\prime}$. It is not hard to see that this probability is $\Theta(1)$ in general if $x$ is in block $\left(k^{\prime}\right)$, and $\Theta\left(1 / n^{4 / 5}\right)$ otherwise (i.e. when $x$ is in block $(t)$ for some $\left.t>k^{\prime}\right)$. Thus by $L=\Theta\left(n^{2 / 5}\right)$ we have

$$
Q L S\left([n]^{2}\right)=\Omega\left(n^{2 / 5} /\left(1+n^{2 / 5} / \sqrt{n^{4 / 5}}\right)\right)=\Omega\left(n^{2 / 5}\right)
$$

This completes the proof of Theorem 3 .

Note that this random walk suffers from the fact that the "passing probability" is now $n^{4 / 5}$ times the "hitting probability". So for general $d$, we can get $R L S\left([n]^{d}\right)=\Omega\left(n^{d /(d+1)}\right)$ and $Q L S\left([n]^{d}\right)=$ $\Omega\left(n^{d /(2 d+1)}\right)$, which only gives better results for $Q L S$ on the 2-dimensional grid.

\section{New algorithms for Local Search on general graphs}

In $[4,2]$, a randomized and a quantum algorithm for Local Search on general graphs are given as follows. Pick $k$ random samplings over all the vertices, and find a vertex $v$ in them with the minimum $f$-value. ${ }^{7}$ Then roughly speaking, $v$ is the $N / k$-minimum vertex over all the $N$ vertices in $G$. Now we follow a decreasing path as follows. Find a neighbor of $v$ with the minimum $f$-value, and continue this minimum-value-neighbor search process until getting to a local minimum. Since $v$ is the $N / k$-minimum vertex, any decreasing path from it has length no more than $N / k$. Thus we need $k+\delta N / k$ queries in the randomized case and $\sqrt{k}+\sqrt{\delta} N / k$ queries in the quantum case, and optimizing $k$ achieves the performance of the algorithms mentioned in Section 1. We can see that the algorithms actually fall into the generic algorithm category (see Section 1), with the initial point picked as the best one over some random samples.

In this section, we give new randomized and quantum algorithms, which work better than this simple "random sampling + steepest descent" method when the graph expands slowly. Here the idea is that after finding the minimum vertex $v$ of the $k$ sampled points, we know that $v$ is (roughly) the $N / k$-minimum vertex. Therefore, there must be a local optimum within the smaller range $\left\{u: l_{G}(u, v) \leq N / k\right\}$. So instead of following the decreasing path of $v$, we do the local search within this smaller range recursively.

While this idea sounds simple and effective, there is one caveat here: a local minimum $u$ in the smaller range may be not a local minimum in the original larger graph $G$, because $u$ may have more neighbors in $G$. To deal with this difficulty, we will actually solve a stronger version of the local search problem: on the graph $G$, given a function $f: V \rightarrow \mathbb{N}$ and a vertex $v$, find a local optimum $u$ s.t. $f(u) \leq f(v)$. Note that such $u$ must exist; any decreasing path from $v$ leads to a valid $u$. Also note that this problem is harder than the original local search problem; any algorithm for this new problem is also an algorithm for the original one. A key property of this new problem is that it allows a recursion: given a small range $S_{\text {small }}\left(\subseteq S_{\text {large }} \subseteq V\right)$ and a vertex $v$ in it, suppose that any vertex $w$ on the boundary ${ }^{8}$ of $S_{\text {small }}$ is worse than $v(i . e . f(w)>f(v)$ ), then any local optimum $u$ in $S_{\text {small }}$ satisfying $f(u) \leq f(v)$ is also a local optimum in a larger range $S_{\text {large }}$.

Now we describe the algorithm precisely, with some notations as follows. For $G=(V, E)$, a given function $f: V \rightarrow \mathbb{N}$, a vertex $v \in V$ and a set $S \subseteq V$, let $n(v, S)=|\{u \in S: f(u)<f(v)\}|$. The boundary $B(S)$ of the set $S \subseteq V$ is defined by $B(S)=\{u \in S: \exists v \in V-S$ s.t. $(u, v) \in E\}$. In particular, $B(V)=\emptyset$. A decreasing path from a vertex $v \in V$ is a sequence of vertices $v_{0}, v_{1}, \ldots, v_{k}$ such that $v_{0}=v, v_{k}$ is a local minimum and $f\left(v_{i+1}\right)=\min _{v:\left(v_{i}, v\right) \in E} f(v)<f\left(v_{i}\right)$ for $i=0, \ldots, k-1$. We write $f(u) \leq f(S)$ if $f(u) \leq f(v)$ for all $v \in S$. In particular, it always holds that $f(u) \leq f(\emptyset)$. Suppose $d=\max _{u, v \in V} l(u, v)$ is the diameter of the graph, and $\delta=\max _{v \in V}|\{u:(u, v) \in E\}|$ is the

\footnotetext{
${ }^{7}$ For the minimum $f$-value finding procedure, The randomized algorithm in [4] just queries all these vertices and find the minimum, while the quantum algorithm in [2] uses the algorithm by Durr and Hoyer [11] based on Grover search [12] to get a quadratic speedup.

${ }^{8}$ The boundary contains those vertices in $S_{\text {small }}$ that have neighbors in $S_{\text {large }}-S_{\text {small }}$.
} 
max degree of the graph. In the following algorithm, the asymptotical numbers at the end of some command lines are the numbers of randomized or quantum queries needed for the step. For those commands without any number, no query is needed.

1. $m_{0}=d, U_{0}=V(G)$;

2. $i=0$;

3. while $\left(\left|m_{i}\right|>10\right)$ do

(a) Randomly pick (with replacement) $\left\lceil\frac{8\left|U_{i}\right|}{m_{i}} \log \frac{1}{\epsilon_{1}}\right\rceil$ vertices from $U_{i}$, where $\epsilon_{1}=1 /\left(10 \log _{2} d\right.$ );

(b) Search the sampled vertices for one $v_{i}$ with the minimal $f$ value.

- Randomized algorithm: query all the sampled vertices and get $v_{i} . \quad-O\left(\frac{8\left|U_{i}\right|}{m_{i}} \log \frac{1}{\epsilon_{1}}\right)$

- Quantum algorithm: use Durr and Hoyer's algorithm [11] with the error probability at most $\epsilon_{2}=1 /\left(10 \log _{2} d\right) . \quad-O\left(\sqrt{\frac{8\left|U_{i}\right|}{m_{i}} \log \frac{1}{\epsilon_{1}}} \log \frac{1}{\epsilon_{2}}\right)$

(c) if $i=0$, then $u_{i+1}=v_{i}$;

else if $f\left(u_{i}\right) \leq f\left(v_{i}\right)$, then $u_{i+1}=u_{i}$;

else $u_{i+1}=v_{i}$;

(d) for $j=1,2, \ldots$

i. Randomly pick $m_{i j} \in M_{i}=\left\{m: m_{i} / 8 \leq m \leq m_{i} / 2,|W(m)| \leq 10\left|U_{i}\right| / m_{i}\right\}$, where $W(m)=\left\{w \in U_{i}: l\left(w, u_{i+1}\right)=m\right\}$. Let $W_{i j}=W\left(m_{i j}\right)$.

ii. Test whether $f\left(u_{i+1}\right) \leq f\left(W_{i j}\right)$

- Randomized algorithm: query all vertices in $W_{i j}$. $\quad-O\left(\left|W_{i j}\right|\right)$

- Quantum algorithm: use Durr and Hoyer's algorithm [11] on $W_{i j}$ with the error probability at most $\epsilon_{3}=1 /\left(200 \log _{2} d\right) . \quad-O\left(\sqrt{\left|W_{i j}\right|} \log \frac{1}{\epsilon_{3}}\right)$

iii. If the answer is Yes, jump out of this for loop and go to Step 3e.

(e) $J_{i}=j, m_{i+1}=m_{i j}, W_{i}=W_{i j}, U_{i+1}=\left\{u \in U_{i}: l\left(u, u_{i+1}\right) \leq m_{i+1}\right\}$;

(f) $i=i+1$;

4. $I=i$;

5. Follow a decreasing path of $u_{I}$ to find a local minimum.

- Randomized algorithm: in each step, query all the neighbors $-O(\delta)$

- Quantum algorithm: in each step, use Durr and Hoyer's algorithm with the error probability at most $1 / 100-O(\sqrt{\delta})$

Define $c(k)=\max _{v \in V}|\{u: l(u, v) \leq k\}|$. Clearly, the expanding speed of a graph is upper bounded by $c(k)$. The following theorem says that the algorithm is efficient if $c(k)$ is small.

Theorem 12 The algorithm outputs a local minimum with probability at least 1/2. The randomized algorithm uses $O\left(\sum_{i=0}^{I-1} \frac{c\left(m_{i}\right)}{m_{i}} \log \log d\right)$ queries in expectation, and the quantum algorithm uses $O\left(\sum_{i=0}^{I-1} \sqrt{\frac{c\left(m_{i}\right)}{m_{i}}}(\log \log d)^{1.5}\right)$ queries in expectation.

In case that $c(k)=O\left(k^{\alpha}\right)$ ( $\alpha$ may be a function of $n$ ) for some $\alpha \geq 1$ and $k=1, \ldots, d$, the expected number of queries that the randomized algorithm uses is $O\left(\frac{d^{\alpha-1}-1}{1-2^{1-\alpha}} \log \log d\right)$ if $\alpha>1$ and $O(\log d \log \log d)$ if $\alpha=1$. The expected number of queries that the quantum algorithm use is $O\left(\frac{d^{\frac{\alpha-1}{2}}-1}{1-2^{\frac{1-\alpha}{2}}}(\log \log d)^{1.5}\right)$ if $\alpha>1$ and $O(\log d \log \log d)$ if $\alpha=1$.

Several comments before proving the theorem:

1. $\lim _{\alpha \rightarrow 1} \frac{d^{\alpha-1}-1}{1-2^{1-\alpha}}=\lim _{\alpha \rightarrow 1} \frac{d^{\frac{\alpha-1}{2}}-1}{1-2^{\frac{1-\alpha}{2}}}=\log _{2} d$ 
2. If $\alpha-1 \geq \epsilon$ for some constant $\epsilon>0$, then $\frac{d^{\alpha-1}-1}{1-2^{1-\alpha}}=\Theta\left(d^{\alpha-1}\right)$ and $\frac{d^{\frac{\alpha-1}{2}}-1}{1-2^{\frac{1-\alpha}{2}}}=\Theta\left(d^{(\alpha-1) / 2}\right)$.

If further the bound $c(k)=O\left(k^{\alpha}\right)$ is tight in the sense that $N=c(d)=\Theta\left(d^{\alpha}\right)$, then $R L S(G)=$ $O\left(\frac{N}{d} \log \log d\right)$ and $Q L S(G)=O\left(\sqrt{\frac{N}{d}}(\log \log d)^{1.5}\right)$.

3. For 2-dimensional grid, $d=\Theta(n)$ and $\alpha=2$. Thus Theorem 5 follows immediately.

Proof We shall prove the theorem for the quantum algorithm. The analysis of the randomized algorithm is almost the same (and actually simpler). We say $W_{i}$ is good if $f\left(u_{i+1}\right) \leq f\left(W_{i}\right)$. We shall first prove the following claim; the theorem then follows easily.

Claim 3 For each $i=0,1, \ldots, I-1$, the following three statements hold.

1. $n\left(u_{i+1}, U_{i+1}\right) \leq n\left(u_{i+1}, U_{i}\right) \leq m_{i} / 8 \leq m_{i+1}$ with probability $1-\epsilon_{1}-\epsilon_{2}$.

2. If $n\left(u_{i+1}, U_{i}\right) \leq m_{i} / 8$, then $W_{i}$ is good with probability $1-\epsilon_{3} J_{i}$, and $\mathbf{E}\left[J_{i}\right] \leq 2$. $^{9}$

3. If $W_{0}, \ldots, W_{i}$ are all good, then $f\left(u_{i+1}\right) \leq f\left(B\left(U_{i+1}\right)\right)$, and $u_{i+1} \notin B\left(U_{i+1}\right)$.

Proof 1: In Step 3a - 3c, denote by $S$ the set of the $\left\lceil\frac{8\left|U_{i}\right|}{m_{i}} \log \frac{1}{\epsilon_{1}}\right\rceil$ sampled vertices in Step 3a. Let $a=\min _{u \in S} f(u)$, then $\left|\left\{v \in U_{i}: f(v)<a\right\}\right| \leq m_{i} / 8$ with probability at least $1-\epsilon_{1}$. The $v_{i}$ found in Step $3 \mathrm{~b}$ achieves the minimum in the definition of $a$ with probability at least $1-\epsilon_{2}$. Put the two things together, we have $n\left(v_{i}, U_{i}\right) \leq m_{i} / 8$ with probability at least $1-\epsilon_{1}-\epsilon_{2}$. Since $f\left(u_{i+1}\right) \leq f\left(v_{i}\right)$ (by Step 3c), $U_{i+1} \subseteq U_{i}$ (by Step 3e) and $m_{i+1} \geq m_{i} / 8$ (by Step 3(d)i), we have $n\left(u_{i+1}, U_{i+1}\right) \leq n\left(u_{i+1}, U_{i}\right) \leq n\left(v_{i}, U_{i}\right) \leq m_{i} / 8 \leq m_{i+1}$ with probability at least $1-\epsilon_{1}-\epsilon_{2}$.

2: We say an $m_{i j}$ is good if the corresponding $W_{i j}$ is good, i.e. $f\left(u_{i+1}\right) \leq f\left(W_{i j}\right)$. Note that for any $m_{i j} \in\left[m_{i}\right]$, we have $W_{i j} \subseteq U_{i}$, and also have $W_{i j} \cap W_{i j^{\prime}}=\emptyset$ if $m_{i j} \neq m_{i j^{\prime}}$. Therefore, if $n\left(u_{i+1}, U_{i}\right) \leq m_{i} / 8$, then at most $m_{i} / 8$ distinct $m_{i j}$ 's in $\left[m_{i}\right]$ are not good. Also note that the number of distinct $m_{i j}$ 's s.t. $\left|W\left(m_{i j}\right)\right|>10\left|U_{i}\right| / m_{i}$ is less than $m_{i} / 10$. Therefore, $\left|M_{i}\right| \geq$ $\left(\frac{3}{8}-\frac{1}{10}\right) m_{i}>m_{i} / 4$. So if $n\left(u_{i+1}, U_{i}\right) \leq m_{i} / 8$, a random $m_{i j}$ in $M_{i}$ is good with probability at least $1 / 2$, and thus $\mathbf{E}\left[J_{i}\right] \leq 2$. Also the probability that all the Grover searches in Step 3(d)ii are correct is at least $1-J_{i} \epsilon_{3}$.

3: We shall first prove $B\left(U_{i+1}\right) \subseteq B\left(U_{i}\right) \cup W_{i}$. In fact, any $s \in B\left(U_{i+1}\right)$ satisfies that $s \in U_{i+1}$ and that $\exists t \in V-U_{i+1}$ s.t. $l(s, t)=1$. Recall that $U_{i+1} \subseteq U_{i}$, so if $t \in V-U_{i}$, then $s \in B\left(U_{i}\right)$ by definition. Otherwise $t \in U_{i}-U_{i+1}$, and thus $t \in U_{i}$ and $l\left(t, u_{i+1}\right)>m_{i+1}$ by the definition of $U_{i+1}$. Noting that $l\left(s, u_{i+1}\right) \leq m_{i+1}$ since $s \in U_{i+1}$, and that $l(s, t)=1$, we have $l\left(s, u_{i+1}\right)=m_{i+1}$, which means $s \in W_{i}$. Thus for all $s \in B\left(U_{i+1}\right)$, either $s \in B\left(U_{i}\right)$ or $s \in W_{i}$ holds, which implies $B\left(U_{i+1}\right) \subseteq B\left(U_{i}\right) \cup W_{i}$.

Applying the result recursively, we have $B\left(U_{i+1}\right) \subseteq B\left(U_{0}\right) \cup W_{0} \cup \ldots \cup W_{i}=W_{0} \cup \ldots \cup W_{i}$. Since we have $f\left(u_{i+1}\right) \leq f\left(u_{i}\right) \leq \ldots \leq f\left(u_{1}\right)$ (by Step 3c) and $f\left(u_{k+1}\right) \leq f\left(W_{k}\right)$ (for $\left.k=0, \ldots, i\right)$ by the assumption that all $W_{k}$ 's are good, we know that $f\left(u_{i+1}\right) \leq f\left(W_{0} \cup \ldots \cup W_{i}\right)$, which implies $f\left(u_{i+1}\right) \leq f\left(B\left(U_{i+1}\right)\right)$.

For the other goal, $u_{i+1} \notin B\left(U_{i+1}\right)$, it is sufficient to prove $u_{i+1} \notin B\left(U_{i}\right)$ and $u_{i+1} \notin W_{i}$. The latter is easy to see by the definition of $W_{i}$. For the former, we can actually prove $u_{k+1} \notin B\left(U_{k}\right)$ for all $k=0, \ldots, i$ by induction on $k$. The base case of $k=0$ is trivial because $B\left(U_{0}\right)=\emptyset$. Now suppose $u_{k} \notin B\left(U_{k-1}\right)$. There are two cases of $u_{k+1}$ by Step 3c. If $f\left(u_{k}\right) \leq f\left(v_{k}\right)$, then $u_{k+1}=u_{k} \notin B\left(U_{k-1}\right)$ by induction. Again by the definition of $W_{k-1}$ we know that $u_{k} \notin W_{k-1}$ and thus $u_{k+1}=u_{k} \notin B\left(U_{k}\right)$. The other case is $f\left(u_{k}\right)>f\left(v_{k}\right)$, then $u_{k+1}=v_{k}$, and therefore $f\left(u_{k+1}\right)=f\left(v_{k}\right)<f\left(u_{k}\right) \leq f\left(B\left(U_{k}\right)\right.$ ) (by the first part in 3), which implies that $u_{k+1} \notin B\left(U_{k}\right)$.

(Continue the proof of Theorem 12) Now by the claim, we know that with probability at least $1-I\left(\epsilon_{1}+\epsilon_{2}\right)-\sum_{i=0}^{I-1} J_{i} \epsilon_{3}$, we will have

$$
n\left(u_{I}, U_{I}\right) \leq m_{I}, \quad f\left(u_{I}\right) \leq f\left(B\left(U_{I}\right)\right), \quad u_{I} \notin B\left(U_{I}\right) .
$$

\footnotetext{
${ }^{9}$ Since $J_{i}$ is a random variable, the meaning of " $W_{i}$ is good with probability $1-\epsilon_{3} J_{i}$ " is that for each fixed $j=1,2, \ldots$, we have that $W_{i}$ is good with probability $1-\epsilon_{3} j$ under the condition that $J_{i}=j$. Similar language is also used in the later part of the proof. Finally we will upper bound the probability of these random variables being large, and in the case that they are small, the error probability is small. This implies that the total error probability is small.
} 
Note that the correctness of the algorithms follows from these three items. Actually, by the last two items, we know that any decreasing path from $u_{I}$ is contained in $U_{I}$. Otherwise suppose $\left(u_{I}^{0}, u_{I}^{1}, \ldots, u_{I}^{T}\right)$ is a decreasing path from $u_{I}$ (so $u_{I}^{0}=u_{I}$ ), and the first vertex out of $U_{I}$ is $u_{I}^{t}$, then $u_{I}^{t-1} \in B\left(U_{I}\right)$. Since $u_{I}^{0} \notin B\left(U_{I}\right)$, we have $t-1>0$ and thus $f\left(u_{I}^{t-1}\right)<f\left(u_{I}\right)$, contradicting to $f\left(u_{I}\right) \leq f\left(B\left(U_{I}\right)\right)$. Now together with the first item, we know that any decreasing path from $u_{I}$ is no more than $m_{I}$ long. Thus Step 5 will find a local minimum by following a decreasing path.

The error probability of the algorithm is $I\left(\epsilon_{1}+\epsilon_{2}\right)+J \epsilon_{3}+10 / 100$, where $J=\sum_{i=0}^{I-1} J_{i}$. Since $\mathbf{E}[J] \leq 2 I$, we know by Markov inequality that $J<20 I$ with probability at least $9 / 10$. Since $\epsilon_{1}=\epsilon_{2}=1 /\left(10 \log _{2} d\right)$ and $\epsilon_{3}=1 /\left(200 \log _{2} d\right)$, and noting that $I \leq \log _{2} d$ because $m_{0}=d$ and $m_{i+1} \leq\left\lceil m_{i} / 2\right\rceil$, the total error probability is less than $1 / 2$.

We now consider the number of queries used in the $i$-th iteration. Note from Step 1 and Step 3e that $\left|U_{i}\right| \leq c\left(m_{i}\right)$ for $i=0,1, \ldots, I-1$. So Step $3 \mathrm{~b}$ uses

$$
O\left(\sqrt{\frac{8\left|U_{i}\right|}{m_{i}} \log \log d} \log \log d\right)=O\left(\sqrt{\frac{c\left(m_{i}\right)}{m_{i}}}(\log \log d)^{1.5}\right)
$$

queries. Also note from Step 3(d)i that $\left|W_{i j}\right| \leq 10\left|U_{i}\right| / m_{i}$, so Step 3d uses $O\left(\sum_{j=1}^{J_{i}} \sqrt{c\left(m_{i}\right) / m_{i}} \log \log d\right)$ queries, which has the expectation of $O\left(\sqrt{c\left(m_{i}\right) / m_{i}} \log \log d\right)$. Finally, Step 5 uses $O(\sqrt{\delta})$ queries. Note that $\delta=c(1)=O\left(c\left(m_{I}\right) / m_{I}\right)$ where $m_{I}$ is a constant integer in the range [6,10]. Altogether, the total expected number of queries used is

$$
O\left(\left(\sum_{i=0}^{\log _{2} d-1} \sqrt{c\left(m_{i}\right) / m_{i}}\right)(\log \log d)^{1.5}\right) .
$$

If $c(k)=O\left(k^{\alpha}\right)$ for some $\alpha \geq 1$ and $k=1, \ldots, d$, then

$$
\sum_{i=0}^{\log _{2} d-1} \sqrt{\frac{c\left(m_{i}\right)}{m_{i}}}=\sum_{i=0}^{\log _{2} d-1} m_{i}^{(\alpha-1) / 2}=\sum_{i=0}^{\log _{2} d-1}\left(d / 2^{i}\right)^{(\alpha-1) / 2}=\frac{d^{\beta}-1}{1-2^{-\beta}}
$$

where $\beta=(\alpha-1) / 2$. This completes the proof for the quantum algorithm, except that in the case of $\alpha=1$ we only have a quantum upper bound of $O\left(\log d(\log \log d)^{1.5}\right)$. But note that the randomized algorithm uses $O(\log d \log \log d)$ queries (because of the saving at error probability controls). So when $\alpha=1$, the quantum algorithm just uses the randomized one.

\section{Open problems: remaining gaps}

We list those grids on which the query complexities of Local Search still have gaps.

\begin{tabular}{|c|c|c|}
\hline$d=$ & 2 & 3 \\
\hline old RLS & {$[\Omega(1), O(n)]$} & {$\left[\tilde{\Omega}(\sqrt{n}), O\left(n^{\frac{3}{2}}\right)\right]$} \\
\hline new RLS & {$\left[\Omega\left(n^{\frac{2}{3}}\right), O(n)\right]$} & {$\left[\Omega\left(n^{\frac{2}{3}} /(\log n)^{\frac{1}{2}}\right), O\left(n^{\frac{3}{2}}\right)\right]$} \\
\hline remaining gap & $n^{\frac{1}{3}}\left(=N^{\frac{1}{6}}\right)$ & $(\log n)^{\frac{1}{2}}\left(=(\log N)^{\frac{1}{2}}\right)$ \\
\hline
\end{tabular}

\begin{tabular}{|c|c|c|c|c|}
\hline$d=$ & 2 & 3 & 4 & 5 \\
\hline old QLS & {$\left[\Omega\left(n^{\frac{1}{4}}\right), O\left(n^{\frac{2}{3}}\right)\right]$} & {$\left[\tilde{\Omega}\left(n^{\frac{1}{4}}\right), O(n)\right]$} & {$\left[\tilde{\Omega}\left(n^{\frac{1}{2}}\right), O\left(n^{\frac{4}{3}}\right)\right]$} & {$\left[\tilde{\Omega}\left(n^{\frac{3}{4}}\right), O\left(n^{\frac{5}{3}}\right)\right]$} \\
\hline new QLS & {$\left[\Omega\left(n^{\frac{2}{5}}\right), O\left(n^{\frac{1}{2}}\right)\right]$} & {$\left[\Omega\left(n^{\frac{3}{4}}\right), O(n)\right]$} & {$\left[\Omega\left(n^{\frac{6}{5}}\right), O\left(n^{\frac{4}{3}}\right)\right]$} & {$\left[\Omega\left(n^{\frac{5}{3}} /(\log n)^{\frac{1}{3}}\right), O\left(n^{\frac{5}{3}}\right)\right]$} \\
\hline remaining gap & $n^{\frac{1}{10}}\left(=N^{\frac{1}{20}}\right)$ & $n^{\frac{1}{4}}\left(=N^{\frac{1}{12}}\right)$ & $n^{\frac{2}{15}}\left(=N^{\frac{1}{30}}\right)$ & $(\log n)^{\frac{1}{3}}\left(=(\log N)^{\frac{1}{3}}\right)$ \\
\hline
\end{tabular}

Here $N=n^{d}$ is the number of the vertices in the grid.

\section{Acknowledgement}


The author thanks Scott Aaronson, Xiaoming Sun and Andy Yao very much for many valuable discussions. Thanks also to Alexander Razborov and Nicholas Pippenger for carefully reading the manuscript and giving many detailed suggestions, to Sean Hallgren, Martin Roetteler, and Pranab Sen for listening to a presentation of the work and giving useful comments. The author is also indebted to Yves Verhoeven and Dirk Winkler for each pointing out an error in a preliminary version of the paper.

\section{References}

[1] K. Aardal, S. Hoesel, J.K. Lenstra, L. Stougie. A Decade of Combinatorial Optimization. CWI Tracts 122, pp. 5-14, 1997.

[2] S. Aaronson. Lower Bounds for Local Search by Quantum Arguments. Proceedings of the thirtysixth Annual ACM Symposium on Theory of Computing, pp. 465-474, 2004.

[3] E. Aarts and J. Lenstra, John Wiley \& Sons, Inc. New York, NY, USA, 1997.

[4] D. Aldous. Minimization Algorithms and Random Walk on the $d$-Cube. Annals of Probability, 11(2), pp. 403-413, 1983.

[5] I. Althofer and K. Koschnich. On the Deterministic Complexity of Searching Local Maxima. Discrete Applied Mathematics 43, pp. 111-113, 1993.

[6] A. Ambainis. Polynomial Degree vs. Quantum Query Complexity. Proceedings of the 44th Annual IEEE Symposium on Foundations of Computer Science, pp. 230-239, 2003.

[7] A. Ambainis. Quantum Lower Bounds by Quantum Arguments, Journal of Computer and System Sciences, 64, pp. 750-767, 2002.

[8] H. Barnum, M. Saks, M. Szegedy. Quantum Query Complexity and Semidefinite Programming. Proceedings of the 18th Annual IEEE Conference on Computational Complexity, pp. 179-193, 2003.

[9] R. Beals, H. Buhrman, R. Cleve, M.Mosca, R. de Wolf. Quantum Lower Bounds by Polynomials. Journal of ACM, 48, pp. 778-797, 2001.

[10] H. Buhrman and R. de Wolf. Complexity Measures and Decision Tree Complexity: a survey. Theoretical Computer Science, Volume 288, Issue 1, pp. 21-43, 2002.

[11] C. Durr and P. Hoyer. A Quantum Algorithm for Finding the Minimum, quant-ph/9607014, 1996.

[12] L. Grover. A Fast Quantum Mechanical Algorithm for Database Search, Proceedings of the 28th Annual ACM Symposium on the Theory of Computing, pp. 212-219, 1996.

[13] P. Hoyer and R. Spalek. Lower Bounds on Quantum Query Complexity, Bulletin of the European Association for Theoretical Computer Science 87, pp. 78-103, 2005.

[14] D. Johnson, C. Papadimitriou, and M. Yannakakis. How Easy is Local Search, Journal of Computer and System Sciences 37, pp. 429448, 1988.

[15] S. Laplante and F. Magniez. Lower Bounds for Randomized and Quantum Query Complexity Using Kolmogorov Arguments. Proceedings of the 19th Annual IEEE Conference on Computational Complexity, pp. 294-304, 2004.

[16] D. Llewellyn and C. Tovey. Dividing and Conquering the Square. Discrete Applied Mathematics 43, pp. 131-153, 1993.

[17] D. Llewellyn, C. Tovey, M. Trick. Local Optimization on Graphs. Discrete Aplied Mathematics 23, pp. 157-178, 1989. Erratum: 46, pp. 93-94, 1993.

[18] N. Megiddo and C. Papadimitriou. On Total Functions, Existence Theorems, and Computational Complexity. Theoretical Computer Science 81, pp. 317324, 1991.

[19] J. Orlin, A. Punnen, A. Schulz. Approximate Local Search in Combinatorial Optimization. SIAM Journal on Computing, 33(5), pp. 12011214, 2004. 
[20] M. Santha and M. Szegedy. Quantum and Classical Query Complexities of Local Search Are Polynomially Related. Proceedings of the thirty-sixth annual ACM symposium on Theory of computing, pp. 494-501, 2004.

[21] R. Spalek and M. Szegedy. All Quantum Adversary Methods Are Equivalent. Proceedings of 32nd International Colloquium on Automata, Languages and Programming, pp. 1299-1311, LNCS 3580, Lisboa, Portugal, 2005.

[22] Y. Verhoeven, Enhanced Algorithms for Local Search. Information Processing Letters 97, pp. $171176,2006$.

[23] S. Zhang. On the Power of Ambainis Lower Bounds, Theoretical Computer Science, 339(2-3), pp. 241-256, 2005. 